\title{
Targeting Toll-Like Receptor 2: Polarization of Porcine Macrophages by a Mycoplasma-Derived Pam2cys Lipopeptide
}

\author{
Giulia Franzoni ${ }^{1, *(\mathbb{C})}$, Antonio Anfossi ${ }^{2}\left(\mathbb{0}\right.$, Chiara Grazia De Ciucis ${ }^{3}$, Samanta Mecocci ${ }^{4}\left(\mathbb{D}\right.$, Tania Carta ${ }^{1,2}$, \\ Silvia Dei Giudici ${ }^{1}{ }^{\mathbb{D}}$, Floriana Fruscione ${ }^{3}$, Susanna Zinellu ${ }^{1}$, Guendalina Vito ${ }^{3}$, Simon Paul Graham ${ }^{5}{ }^{(0)}$, \\ Annalisa Oggiano ${ }^{1}\left(\mathbb{D}\right.$, Bernardo Chessa ${ }^{2, *}$ and Elisabetta Razzuoli ${ }^{3}(\mathbb{B}$
}

1 Department of Animal Health, Istituto Zooprofilattico Sperimentale della Sardegna, 07100 Sassari, Italy; tania.carta@izs-sardegna.it (T.C.); Silvia.DeiGiudici@izs-sardegna.it (S.D.G.); Susanna.Zinellu@izs-sardegna.it (S.Z.); Annalisa.Oggiano@izs-sardegna.it (A.O.)

2 School of Veterinary Medicine, University of Sassari, 07100 Sassari, Italy; aanfossi@uniss.it

3 National Reference Center of Veterinary and Comparative Oncology (CEROVEC), Istituto Zooprofilattico Sperimentale del Piemonte, Liguria e Valle d'Aosta, Piazza Borgo Pila 39/24, 16129 Genoa, Italy; chiaragrazia.deciucis@izsto.it (C.G.D.C.); floriana.fruscione@izsto.it (F.F.); Guendalina.vito@izsto.it (G.V.); elisabetta.razzuoli@izsto.it (E.R.)

4 Department of Veterinary Medicine, University of Perugia, 06123 Perugia, Italy; samanta.mecocci@studenti.unipg.it

5 The Pirbright Institute, Ash Road, Pirbright, Woking GU24 ONF, UK; simon.graham@pirbright.ac.uk

* Correspondence: giulia.franzoni@izs-sardegna.it (G.F.); bchessa@uniss.it (B.C.)

\section{check for} updates

Citation: Franzoni, G.; Anfossi, A.; De Ciucis, C.G.; Mecocci, S.; Carta, T.; Dei Giudici, S.; Fruscione, F.; Zinellu, S.; Vito, G.; Graham, S.P.; et al. Targeting Toll-Like Receptor 2: Polarization of Porcine Macrophages by a Mycoplasma-Derived Pam2cys Lipopeptide. Vaccines 2021, 9, 692. https://doi.org/10.3390/

vaccines 9070692

Academic Editor: Ralph A. Tripp

Received: 17 May 2021

Accepted: 18 June 2021

Published: 23 June 2021

Publisher's Note: MDPI stays neutral with regard to jurisdictional claims in published maps and institutional affiliations.

Copyright: (c) 2021 by the authors. Licensee MDPI, Basel, Switzerland. This article is an open access article distributed under the terms and conditions of the Creative Commons Attribution (CC BY) license (https:// creativecommons.org/licenses/by/ $4.0 /)$.

\begin{abstract}
Toll-like receptor 2 (TLR2) ligands are attracting increasing attention as prophylactic and immunotherapeutic agents against pathogens and tumors. We previously observed that a synthetic diacylated lipopeptide based on a surface protein of Mycoplasma agalactiae (Mag-Pam2Cys) strongly activated innate immune cells, including porcine monocyte-derived macrophages (moM $\Phi)$. In this study, we utilized confocal microscopy, flow cytometry, multiplex cytokine ELISA, and RT-qPCR to conduct a comprehensive analysis of the effects of scalar doses of Mag-Pam2Cys on porcine moMФ. We observed enhanced expression of activation markers (MHC class I, MHC class II DR, CD25), increased phagocytotic activity, and release of IL-12 and proinflammatory cytokines. MagPam2Cys also upregulated the gene expression of several IFN- $\alpha$ subtypes, p65, NOS2, and molecules with antimicrobial activities (CD14, beta defensin 1). Overall, our data showed that Mag-Pam2Cys polarized porcine macrophages towards a proinflammatory antimicrobial phenotype. However, Mag-Pam2Cys downregulated the expression of IFN- $\alpha 3$, six TLRs (TLR3, -4, -5, -7, -8, -9), and did not interfere with macrophage polarization induced by the immunosuppressive IL-10, suggesting that the inflammatory activity evoked by Mag-Pam2Cys could be regulated to avoid potentially harmful consequences. We hope that our in vitro results will lay the foundation for the further evaluation of this diacylated lipopeptide as an immunopotentiator in vivo.
\end{abstract}

Keywords: pig; macrophages; TLR2 agonist; cytokines; surface markers; IFN- $\alpha$ subtypes; toll-like receptors; IL-10

\section{Introduction}

Toll-like receptors (TLRs) are a group of pattern recognition receptors (PRRs), which play critical roles in initiating host immune defenses. They recognize molecules expressed by pathogens, named pathogen-associated molecular patterns (PAMPs), and endogenous ligands (danger-associated molecular patterns or DAMPs). Some TLRs are located on the cell membrane (TLR1, -2, -4, -5, -6) and recognize microbial lipopolysaccharide or lipopeptides, whereas others are localized intracellularly (TLR3, -7, -8, -9) and bind nucleic acids $[1,2]$. TLR-ligands are attracting increasing attention as prophylactic and/or therapeutic agents against infectious diseases [3] or in cancer immunotherapy [4]. These molecules target the host rather than pathogens; thus, they are characterized by a broad spectrum 
of activity and low risk for development of antimicrobial resistance. TLR-agonists can also be used in combination with vaccines as molecular adjuvants, being able to provide a "danger" signal to help induce effective and long-lasting adaptive immune responses [2].

Upon PAMP recognition by TLRs, several intracellular signaling cascades are triggered, which also culminate in inflammasome activation, with consequent inflammatory responses that limit invading pathogens' progression $[5,6]$. However, a tight regulation of this process is essential not only to achieve pathogen clearance, but also to avoid pathogenic inflammatory responses [6]. TLR-2 agonists are amongst the TLR-ligands that have shown promise against both pathogens [3] and tumors [7]. The well-characterized macrophage-activating lipopeptide-2 (MALP-2), a lipopeptide originating from Mycoplasma fermentans [8], presented remarkable immunomodulatory properties in vivo: its intratracheal administration in rodents led to a recruitment of neutrophils and macrophages to the lungs and increased protection against Streptococcus pneumonia [9]. A synthetic analog of MALP-2, S-[2,3-bis(palmitoyl oxy)propyl] cysteine (Pam2Cys) is a potent adjuvant that has been incorporated into many vaccine candidates [10-15]. A soluble version of pegylatedPam2Cys (PEG-Pam2Cys) was intranasally administered in mice in the absence of an antigen [14], leading to a significant recruitment of innate immune cells to the lungs, with subsequent protection to challenge influenza A virus [14]. Most recently, it was reported that intranasal administration of another synthetic TLR2/TLR6 agonist (INNA-051) to ferrets resulted in reduced SARS-CoV-2 shedding in the upper respiratory tract [16].

We previously reported a diacylated lipopeptide, chemically synthesized based on the 14 amino acids following the cysteine immediately downstream the signal peptide of a surface protein of M. agalactiae (P48), strongly activated cells of the innate immune system including: ovine neutrophils [17] and monocytes [18], and porcine monocyte-derived macrophages $(\operatorname{moM} \Phi)[19,20]$. This diacylated lipopeptide activates cells upon recognition by TLR2, in association with TLR6 $[3,17]$. We most recently described that this synthetic lipopeptide induced a strong release of IL-12 and proinflammatory cytokines from porcine $\operatorname{moM} \Phi[19,20]$.

Macrophages are a key component of the innate immune system, responding to both infectious and non-infectious stressors [21]. They are the primary target cell of several viruses threatening the swine industry worldwide, such as the African swine fever virus (ASFV), porcine reproductive and respiratory syndrome virus (PRRSV), and porcine circovirus 2 [22-25]. New therapeutics, such as immunopotentiators and vaccine adjuvants, might be able to improve the immunological control of these pathogens and reduce the associated economic losses. In addition, pig macrophages resemble human macrophages in many aspects [26-28], and overall pig and humans share many immunological similarities [29-31], making them an attractive biomedical model [32].

In this study, we deployed an array of techniques to investigate further the effects of Mag-Pam2Cys on porcine macrophages with the aim of informing its potential use as an immunomodulant in vivo.

\section{Materials and Methods}

\subsection{Animals}

Six healthy cross-bred pigs (Sus scrofa domesticus), 6-24 months old, were utilized as blood donors. Animals were kept at the Experiment Station of Istituto Zooprofilattico Sperimentale (IZS) of Sardinia ("Surigheddu", Sassari, Italy). Heparinized blood samples were collected by puncture of the cranial vena cava; all procedures were performed in accordance with the local ethics committee, in agreement with the Guide of Use of Laboratory Animals issued by the Italian Ministry of Health.

\subsection{Generation of Porcine Monocyte-Derived Macrophages and Activation}

MoM $\Phi$ cultures were obtained from blood leukocytes, as previously described [20]. In brief, pig leukocytes were first purified from heparinized blood: pig blood was centrifuged at $700 \times g$ for 30 min at $4{ }^{\circ} \mathrm{C}$ without breaks; then, buffy coat was collected, washed three 
times in red blood cell lysis buffer (distilled water with $0.5 \mathrm{mM} \mathrm{Na}$ EDTA, $310 \mathrm{mM} \mathrm{NH}_{4} \mathrm{Cl}$, $24 \mathrm{mM} \mathrm{NaHCO}$ ), and finally washed once in PBS. Leukocytes were then cultured in RPMI-1640 supplemented with $10 \%$ fetal bovine serum (FBS), $100 \mathrm{U} / \mathrm{mL}$ penicillin, and $100 \mu \mathrm{g} / \mathrm{mL}$ streptomycin (complete RPMI, cRPMI), and with $50 \mathrm{ng} / \mathrm{mL}$ of recombinant human M-CSF (hM-CSF) (Thermo Fisher Scientific, Waltham, MA, USA), using Petri dishes $\left(2 \times 10^{7}\right.$ leukocytes $/ \mathrm{mL} ; 20 \mathrm{~mL}$ per Petri dish). After 7 days, non-adherent leukocytes were removed, and adherent moM $\Phi$ were detached by gentle scraping using a $25 \mathrm{~mL}$ pipette. Cells were centrifuged at $200 \times g$ for $8 \mathrm{~min}$; supernatants were removed, and moM $\Phi$ were finally re-suspended in CRPMI, and seeded in 12-well plates (Greiner CELLSTAR, Sigma-Aldrich, Saint Louis, MO, USA) $\left(1 \times 10^{6}\right.$ live cells per well $)$ or 8 -well chamber slides (Thermo Fisher Scientific) $\left(1 \times 10^{5}\right.$ live cells per well). Cells were incubated at $37^{\circ} \mathrm{C} 5 \%$ $\mathrm{CO}_{2}$ for a further $24 \mathrm{~h}$ before treatment [20].

MoM $\Phi$ were left untreated or stimulated with 10 or $100 \mathrm{ng} / \mathrm{mL}$ of the TLR2 agonist S[2-bis(palmitoyl)-propyl]cysteine (Pam2Cys) lipopeptide (Mag-Pam2Cys). Mag-Pam2Cys was chemically synthesized based on the 14 amino acids following the cysteine immediately downstream the signal peptide of a M. agalactiae lipoprotein (P48: CGDKYFKETEVDGV) (Espikam, Prato, Italy) [17].

In selected experiments, cells were stimulated with $100 \mathrm{ng} / \mathrm{mL}$ of recombinant LPS (lipopolysaccharide from Escherichia coli 0111:B4; Sigma-Aldrich) and $100 \mathrm{ng} / \mathrm{mL}$ of recombinant porcine IFN- $\gamma$ (RayBiotech Inc., Norcross, GA, USA) for $24 \mathrm{~h}$, in order to generate moM1, as previously described [20].

In other selected experiments, cells were stimulated with Mag-Pam2Cys (10 or $100 \mathrm{ng} / \mathrm{mL})$ and recombinant porcine IL-10 (20 ng/mL). Recombinant porcine IL-10 (R\&D System, Minneapolis, MN, USA) was added simultaneously for $24 \mathrm{~h}$ before Mag-Pam2Cys stimulation. Untreated cells were included as controls.

\subsection{Cell Viability}

The impact of Mag-Pam2Cys on $\operatorname{moM} \Phi$ viability was evaluated using a non-radioactive cytotoxicity assay [33]. In brief, cells were seeded in 12 well plates $\left(1 \times 10^{6}\right.$ live cells per well) and left untreated (negative control) or cultured in the presence of scalar doses of Mag-Pam2Cys (10 and $100 \mathrm{ng} / \mathrm{mL}$ ). Then, $24 \mathrm{~h}$ later, lactate dehydrogenase (LDH) levels in culture supernatants were quantified using the Cytotox 96 Non-Radioactive Cytotoxicity Assay (Promega, Madison, WI, USA), and a lysis solution provided by the manufacturer was used as a positive control, according to the manufacturer's instruction. Absorbance was read at $492 \mathrm{~nm}$ using an Epoch microplate reader (BioTek, Winoosky, VT, USA).

\subsection{Flow Cytometry}

MoM $\Phi$ were seeded in 12 well plates $\left(1 \times 10^{6}\right.$ live cells per well $)$ and stimulated with Mag-Pam2Cys, with or without IL-10. Untreated cells were used as a control. Then, $24 \mathrm{~h}$ post stimulation, flow cytometry was carried out as previously described [19]. In brief, cells were harvested with PBS with $10 \mathrm{mM}$ EDTA and transferred into $5 \mathrm{~mL}$ round bottom tubes (Corning). MoM $\Phi$ were washed, resuspended in $0.1 \mathrm{~mL}$ per tube, and stained with Zombie Aqua dye (BioLegend, San Diego, CA, USA), and after $30 \mathrm{~min}$ of incubation at room temperature, cells were washed with PBS supplemented with $0.5 \%$ bovine serum albumin (BSA). Cells were then resuspended in $0.1 \mathrm{~mL}$ of PBS, and staining with monoclonal antibodies was performed using mouse anti human CD14-PerCP (Tuk4; Miltenyi Biotec, Bergisch Gladbach, Germany), mouse anti pig MHC class II DR-FITC (2E9/13, Bio-Rad Antibodies, Kidlington, UK), MHC class I (JM1E3, Bio-Rad Antibodies), CD163-PE (2A10/11, Bio-Rad Antibodies), CD169-FITC (MCA2316F, Bio-Rad Antibodies), and CD25 (K231.3B2, Bio-Rad Antibodies). Incubation was performed at $4{ }^{\circ} \mathrm{C}$ for $15 \mathrm{~min}$. MHC I and CD25 expression were visualized by subsequent staining with BV421 rat anti mouse IgG1 (A85-1, BD Horizon, BD Biosciences, Franklin Lakes, NJ, USA) for a further $15 \mathrm{~min}$. Both primary and secondary antibody were added at a final volume of $10 \mu \mathrm{L}$ per tube; their details are reported in Table S1. After washing with PBS supplemented with $2 \%$ 
FBS, cells were resuspended in PBS supplemented with $2 \mathrm{mM}$ EDTA and were analyzed with a FACS Celesta (BD Biosciences). A total of 5000 live moM $\Phi$ were acquired. Analysis of data was performed using BD FACS Diva Software (BD Biosciences), by exclusion of doublets, gating on viable $\operatorname{moM} \Phi$, and then assessing the staining for surface markers [19].

\subsection{Impact of Pam2Cys on moMФ Morphology}

MoMФ were cultured in 8-well chamber slides (for confocal microscopy) or 12-well plates (for flow cytometry), at $1 \times 10^{5}$ or $1 \times 10^{6}$ live cells per well, respectively. Then, $24 \mathrm{~h}$ post-activation, Mag-Pam2Cys' impact on moM $\Phi$ phenotype was assessed. For confocal microscopy, moM $\Phi$ were fixed with $4 \%$ paraformaldehyde and then labelled with Hoechst 33342 and Alexa Fluor 488 conjugated phalloidin (both Molecular Probes, Thermo Fisher Scientific, Rockford, IL, USA) to visualize nuclei or actin cytoskeleton, respectively [34]. Confocal microscopy was performed using a Leica SP5 Confocal Microscope (Leica Microsystem, Wetzlar, Germany) equipped with a 40X Plan-Apo 1.25 NA oil immersion objective. Images were acquired on a format of $1024 \times 1024$ pixel and were processed with LAS AF Lite software (Leica Microsystem), as previously described [34]. Phase-contrast microscopy images were also acquired: MoMФ were fixed with $4 \%$ paraformaldehyde, washed with PBS, and then images were acquired using an inverted stereo microscope Olympus IX 70 (Segrate, Italy). Furthermore, moM $\Phi$ were morphologically evaluated though May-Grunwald-Giemsa staining. In brief, moM $\Phi$ were fixed, washed with PBS, and stained using the May-Grünwald-Giemsa method, and images were acquired and recorded using a light microscope (Nikon Eclipse 80i, Tokyo, Japan) equipped with a Nikon Ds-fil camera (Tokyo, Japan). Flow cytometry was carried as above described, and forward scatter (FSC) and side scatter (SSC) data were used to determine effects on the dimension and granularity of moM $\Phi$.

\subsection{Phagocytosis Assay}

MoM $\Phi$ were cultured in 8-well chamber slides $\left(1 \times 10^{5}\right.$ live cells/well). Then, $24 \mathrm{~h}$ after seeding, cells were left untreated or stimulated with Mag-Pam2Cys (10 or $100 \mathrm{ng} / \mathrm{mL}$ ), and phagocytosis assays were performed $24 \mathrm{~h}$ later using pHrodo Red Zymosan A Bioparticles Conjugated (Molecular Probes), following the manufacturer's instruction. In brief, culture supernatants were removed, replaced with $0.1 \mathrm{~mL}$ of pHrodo bioparticles suspension $\left(0.5 \mathrm{mg}\right.$ bioparticles $/ \mathrm{mL}$ ), and incubated for $2 \mathrm{~h}$ at either $4{ }^{\circ} \mathrm{C}$ (control) or $37^{\circ} \mathrm{C}$ for $2 \mathrm{~h}$. МоMФ were then fixed with $4 \%$ paraformaldehyde and labelled with Hoechst 33342 (Molecular Probes) to visualize nuclei. Microscopy was performed as above described, using a Leica SP5 Confocal Microscope, and processing images with LAS AF Lite software [34].

\subsection{Cytokine Levels Determination}

MoM $\Phi$ were cultured in 12-well plates $\left(1 \times 10^{6}\right.$ live cells/well) and stimulated with Mag-Pam2Cys (10 or $100 \mathrm{ng} / \mathrm{mL}$ ) or left untreated. Then, $24 \mathrm{~h}$ later, culture supernatants were removed, cleared by centrifugation $(2000 \times g$ for $3 \mathrm{~min})$, and stored at $-80{ }^{\circ} \mathrm{C}$ until analysis. Levels of IL- $1 \alpha$, IL-1 $\beta$, L-6, IL-10, IL-12, TNF- $\alpha$ were determined using a Porcine Cytokine/Chemokine Magnetic Bead Panel Multiplex assay (Merck Millipore, Darmstadt, Germany) and a Bioplex MAGPIX Multiplex Reader (Bio-Rad, Hercules, CA, USA), according to the manufacturers' instructions, as previously described [34].

\subsection{Gene Expression}

Changes in the mRNA expression profile were evaluated as previously described $[19,35,36]$. In brief, moM $\Phi$ were seeded in 12 well plates $\left(1 \times 10^{6}\right.$ live cells/well $)$ and left untreated or stimulated with Mag-Pam2Cys (10 or $100 \mathrm{ng} / \mathrm{mL})$; at selected time points $(0,4,8,24 \mathrm{~h})$, culture supernatants were removed, and cells were lysed with buffer RTL (Qiagen, Hilden, Germany). Total RNA was then extracted using the RNeasy Mini Kit (Qiagen) and eluted in $100 \mu \mathrm{L}$ of ultrapure RNase-free water. Furthermore, $250 \mathrm{ng}$ of purified RNA was used 
as the template for cDNA synthesis, as previously described [36]. Gene expression was evaluated by RT-qPCR, using primer sets reported in Table S2 [37-41]. Real-time PCR amplification was performed in a CFX96 Real-Time System after the reverse transcription step, with glyceraldehyde 3-phosphate dehydrogenase (GAPDH) as a reference gene [35]. In each sample, the relative expression of the test genes was calculated using the widely adopted $2^{-\Delta \Delta \mathrm{Cq}}$ method, with the $\mathrm{Cq}$ acronym of the quantification cycle [35].

\subsection{Statistical Analysis}

Experiments were carried out in technical duplicate and repeated with at least three different blood donor pigs. Data were first checked for normality using Minitab (Minitab Inc., Coventry, UK), and then they were graphically and statistically analyzed with GraphPad Prism 8.01 (GraphPad Software Inc., La Jolla, CA, USA). Data normality was determined using the Anderson Darling test; then, data were analyzed using the parametric one-way ANOVA followed by Dunnett's multiple comparison test or the non-parametric KruskalWallis test followed by a Dunn's multiple comparison test. In both cases, a $p$ value $<0.05$ was considered statistically significant; $p<0.05\left(^{*}\right), p<0.01\left(^{* *}\right), p<0.001\left(^{* * *}\right)$.

\section{Results}

The ability of Mag-Pam2Cys, a chemically synthesized TLR2 agonist, to modulate the porcine $\operatorname{moM} \Phi$ phenotype and functionality was assessed though an integrative analytical approach, spanning flow cytometry, confocal microscopy, multiplex ELISA, and RT-qPCR. The ability of this lipopeptide to affect the macrophage's responses to the immunosuppressive IL-10 was also evaluated (Figure S1).

\subsection{Impact of Mag-Pam2Cys on moM $\Phi$ Phenotype and Viability}

MoMФ were left untreated or stimulated with 10 or $100 \mathrm{ng} / \mathrm{mL}$ of Mag-Pam2Cys. Then, $24 \mathrm{~h}$ later, their morphology was assessed by confocal microscopy and flow cytometry (Figure 1). Mag-Pam2Cys did not significantly impact moM $\Phi$ morphology: Cells presented with a spherical shape with short hairy protrusions on their surface, as observed in our previous study [19], irrespective of the treatment (Figure 1a). No morphological alterations were observed using phase contrast microscopy or May-Grunwald-Giemsa staining (Figure 1b, Figure S2). No changes in cellular dimension or granularity were detected between Mag-Pam2Cys stimulated or untreated moM $\Phi$ (Figure 1c). At the same time point post-stimulation $(24 \mathrm{~h})$, the effects of Mag-Pam2Cys on moM $\Phi$ viability were quantified using a cytotoxicity assay. A lysis solution provided by the manufacturer and untreated $\operatorname{moM} \Phi$ were used as positive and negative controls, respectively. Mag-Pam2Cys had no appreciable impact on porcine macrophage viability (Figure 1c).

The modulation of several key surface markers by Mag-Pam2Cys was assessed by flow cytometry (Figure 2, Figure S3). Both 10 and $100 \mathrm{ng} / \mathrm{mL}$ doses of Mag-Pam2Cys induced an upregulation of the surface expression of MHC I, MHC II DR, and CD25. Statistically significantly upregulation of MHC II DR and CD25 was appreciated in terms of both mean fluorescence intensity (MFI) and percentage of positive cells, and a significant increase in the MFI (but not percentage of positive cells) of the ubiquitously expressed MHC I was observed (Figure 2, Figure S3). No differences were observed between untreated and Mag-Pam2Cys stimulated moM $\Phi$ in terms of percentages of CD163 ${ }^{+}$or $\mathrm{CD} 169^{+}$cells. Stimulation with this TLR2 agonist induced a slight increase in CD14 expression (MFI), although without statistical significance (Figure 2, Figure S3).

\subsection{Evaluation of the Effects of Mag-Pam2Cys on moM $\Phi$, Phagocytic Capacity, and Cytokine Responses}

The effect of Mag-Pam2Cys on moM $\Phi$ phagocytotic activity was then monitored using confocal microscopy. At $24 \mathrm{~h}$ post-stimulation, an increased number of moM $\Phi$ associated with red-labelled bioparticles was observed in the Mag-Pam2Cys-treated condition compared to the untreated control, especially in cells exposed to the higher dose $(100 \mathrm{ng} / \mathrm{mL})$ (Figure 3). 
a

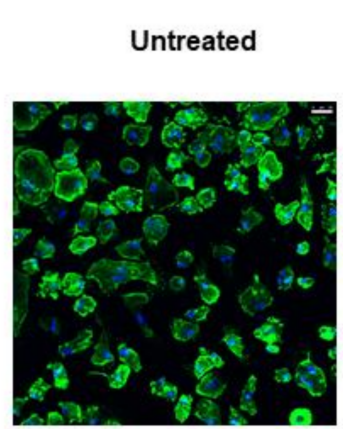

\section{Mag-Pam2Cys (10 $\mathrm{ng} / \mathrm{mL}$ )}

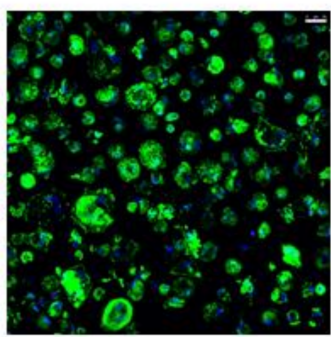

b

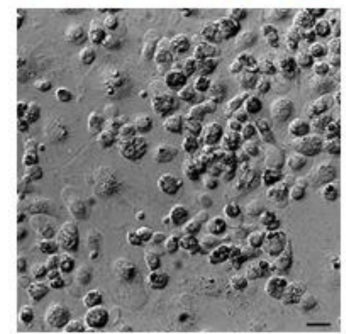

\section{Mag-Pam2Cys} (100 ng/mL)
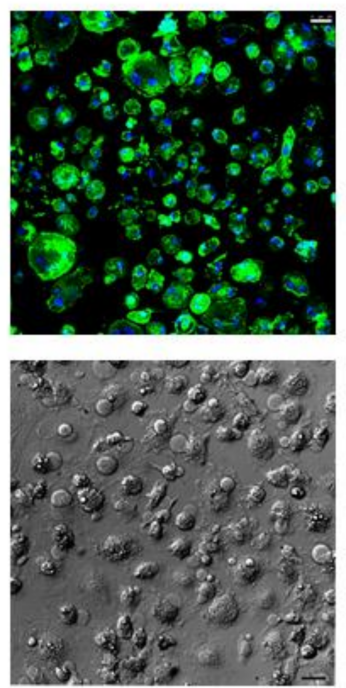

C

Dimension

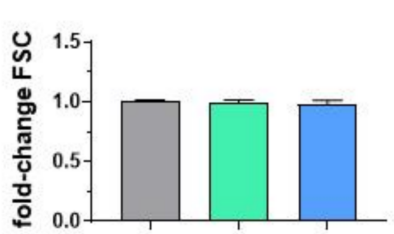

Granularity

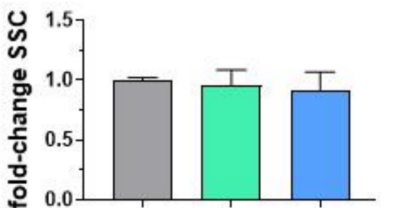

$\square$ Untreated

$10 \mathrm{ng} / \mathrm{ml}$ Mag-Pam2Cys ㅁ $100 \mathrm{ng} / \mathrm{ml}$ Mag-Pam2Cys

d

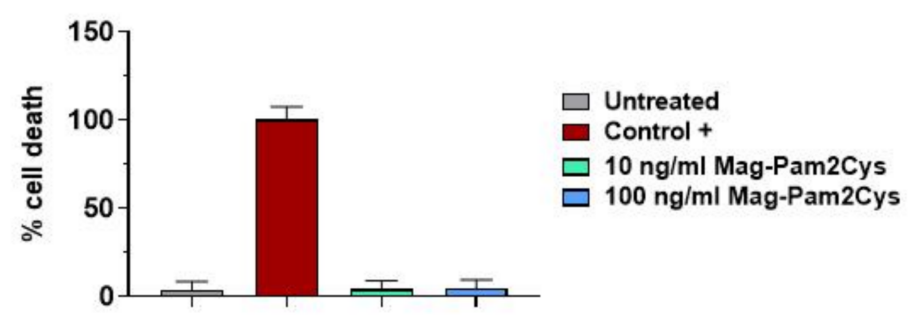

Figure 1. Impact of Mag-Pam2Cys on porcine moM $\Phi$ morphology and viability. Porcine moM $\Phi$ were stimulated with scalar doses of Mag-Pam2Cys (10 or $100 \mathrm{ng} / \mathrm{mL}$ ) or left untreated. Then, 24 h poststimulation, morphology (a-c) and viability (d) were assessed. (a) Confocal microscopy observation after nuclei staining with Hoechst 33342 (blue) and cytoskeleton with Alexa Fluor 488-conjugated phalloidin (green), with magnification $40 \times$. Scale bar, $25 \mu \mathrm{m}$. (b) Phase-contrast microscopy images were acquired using an inverted microscope, with magnification $20 \times$. Scale bar, $25 \mu \mathrm{m}$. For both $a$ and $b$, images of representative $\operatorname{moM} \Phi$, one from each condition (untreated, 10 or $100 \mathrm{ng} / \mathrm{mL}$ of Mag-Pam2Cys) are presented. (c) Changes in dimension and granularity of moM $\Phi$ were evaluated by flow cytometry. Forward scatter (FSC) and side scatter (SSC) data are displayed as fold-change relative to untreated $\operatorname{moM} \Phi$. (d) Mag-Pam2Cys impact on $\operatorname{moM} \Phi$ viability was assessed using a non-radioactive cytotoxic assay. A lysis solution provided by the manufacturer was used as positive control (Control +). For both Panel $(\mathbf{c}, \mathbf{d})$, mean data for triplicate biological replicates and standard deviation (SD) are shown. Values of stimulated samples were compared to the untreated control using a one-way ANOVA followed by a Dunnett's multiple comparison test. 
a

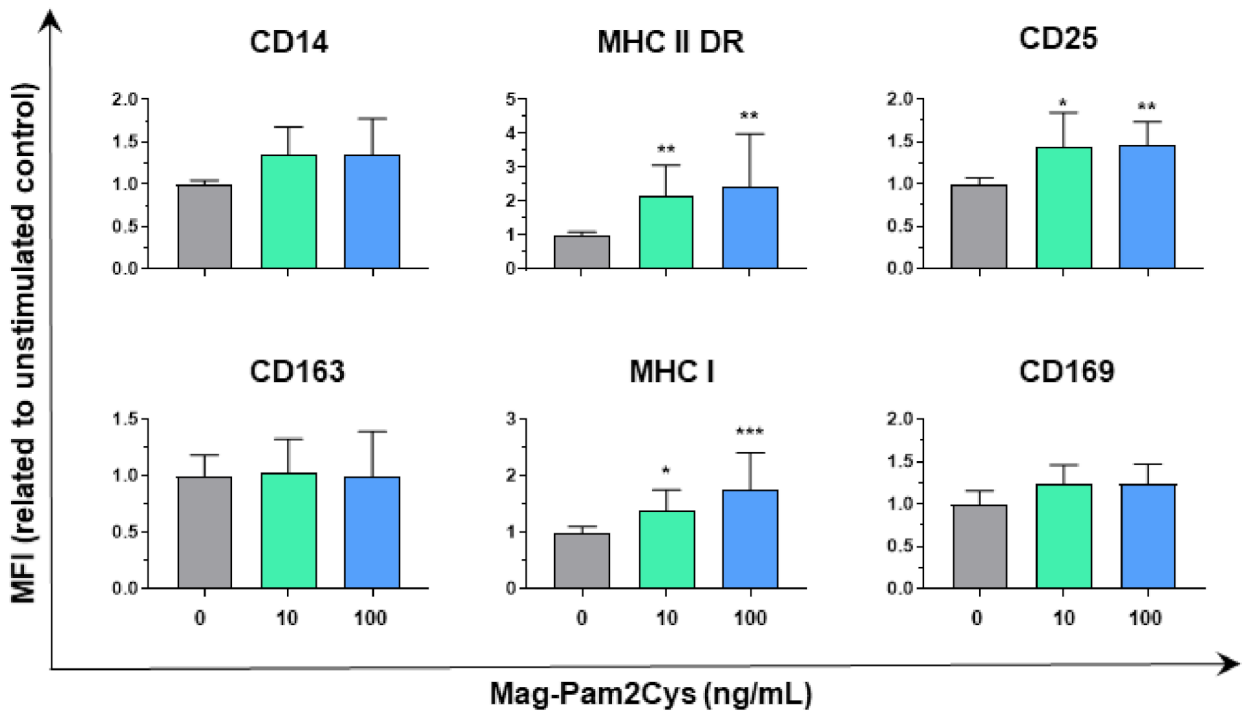

b

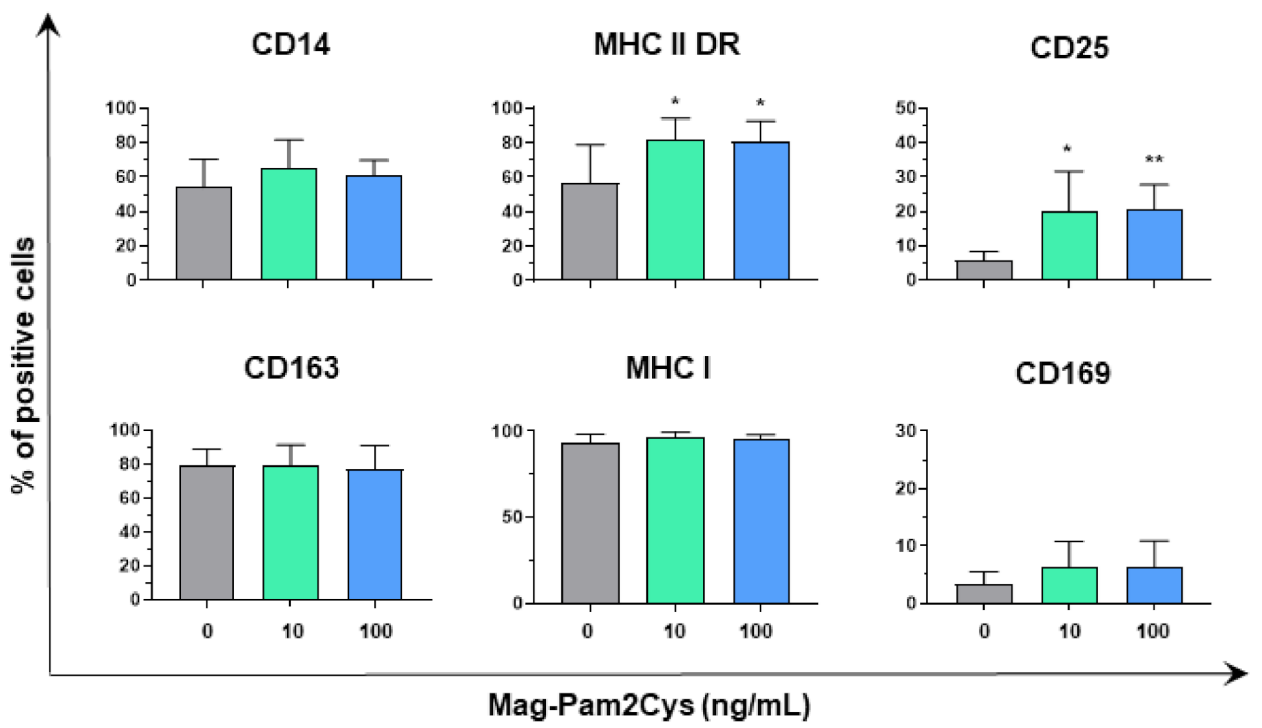

Figure 2. Effect of Mag-Pam2Cys on moM $\Phi$ surface marker expressions. Then, $24 \mathrm{~h}$ post-stimulation with scalar doses of Mag-Pam2Cys (10 or $100 \mathrm{ng} / \mathrm{mL}$ ), surface expression of CD14, MHC II DR, CD25, CD163, MHC I, and CD169 were assessed by flow cytometry. In Panel (a), mean fluorescence intensity (MFI) data are presented as fold-change relative to the untreated control (moM $\Phi)$. In Panel (b), percentages of positive cells are shown. For both Panels, mean data and SD from four independent experiment using different blood donor pigs are displayed. Values of Mag-Pam2Cystreated samples were compared to the untreated control $(\operatorname{moM} \Phi)$ using a one-way ANOVA followed by a Dunnett's multiple comparison test. ${ }^{* * *} p<0.001,{ }^{* *} p<0.01,{ }^{*} p<0.05$. 

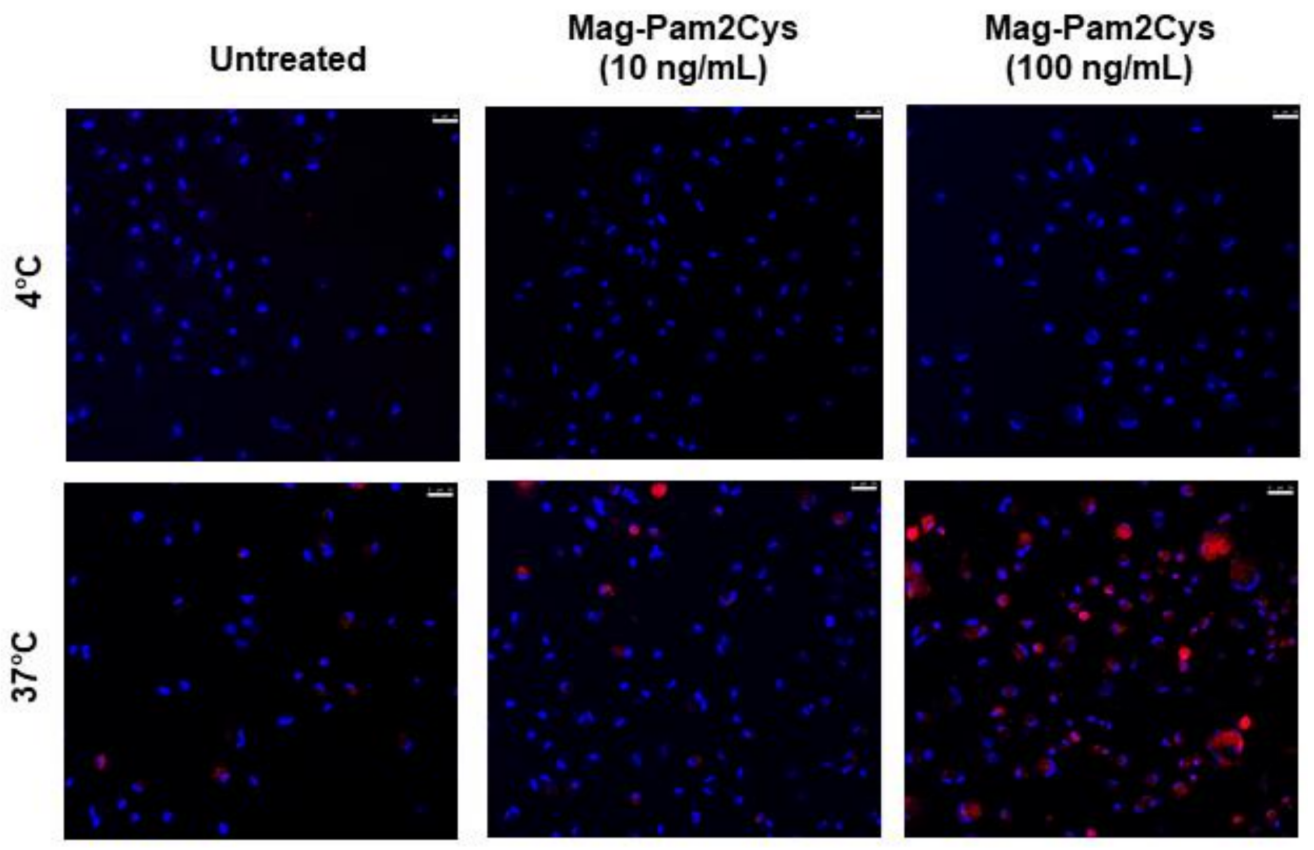

Figure 3. Mag-Pam2Cys' impact on $\operatorname{moM} \Phi$ phagocytotic ability. Porcine moM $\Phi$ were left untreated or stimulated with scalar doses of Mag-Pam2Cys (10 or $100 \mathrm{ng} / \mathrm{mL}$ ). At $24 \mathrm{~h}$ post-stimulation, a phagocytosis assay was performed using pHrodoTM Red Zymosan A Bioparticles Conjugate (red). Cells were incubated with bioparticles for $2 \mathrm{~h}$, at either $4{ }^{\circ} \mathrm{C}$ (control) or $37^{\circ} \mathrm{C}$. Then, nuclei were stained with Hoechst 33342 (blue), and confocal microscopy was performed using a 40× magnification. Images of representative $\mathrm{moM} \Phi$, one from each condition (untreated, $10 \mathrm{or} 100 \mathrm{ng} / \mathrm{mL}$ of Mag-Pam2Cys) are presented. Scale bar, $25 \mu \mathrm{m}$.

At the same time post-stimulation, the cytokine content in culture supernatants was assessed by ELISA. Levels of several proinflammatory cytokines (IL-1 $\alpha$, IL-1 $\beta$, IL-6, TNF- $\alpha$ ) and IL-12 were increased in Mag-Pam2Cys-stimulated cultures (Figure 4a). Low levels of IL10 were also detected in the culture supernatant of cells exposed to either 10 or $100 \mathrm{ng} / \mathrm{mL}$ of Mag-Pam2Cys, although without statistical significance compared to the untreated control (Figure 4a). To investigate $\operatorname{moM} \Phi$ cytokine response to Mag-Pam2Cys further, gene expression analyses of IL- $1 \beta$, IL-6, TNF- $\alpha$, IL-12p40, and IL-10 were performed. MoM $\Phi$ were exposed to Mag-Pam2Cys (10 or $100 \mathrm{ng} / \mathrm{mL}$ ) or left untreated, and at 4, 8, and $24 \mathrm{~h}$ post-stimulation, expression analysis of the five selected cytokine genes was performed (Figure 4b). RT-qPCR data revealed that Mag-Pam2Cys induced enhanced expression of IL-1 $\beta$, IL-6, TNF- $\alpha$, and IL-12p40 at all the tested time points, in full accordance with the cytokine protein levels detected in $\operatorname{moM} \Phi$ culture supernatants. Statistically significant increased expression of IL-10 was observed only at 4 and $8 \mathrm{~h}$ post-stimulation (Figure $4 \mathrm{~b}$, Figure S4).

\subsection{Comparison of Mag-Pam2Cys and M1-Polarization}

So far, our data revealed that Mag-Pam2Cys induced an upregulation of the surface expression of MHC I, MHC II DR, and CD25, increased phagocytic activity, and a release of IL-1 $\beta$, IL-6, IL-12p40, TNF- $\alpha$. These data suggest that Mag-Pam2Cys polarized porcine macrophages toward a M1-like phenotype [34,42]; thus, we compared the impact of the higher dose of Mag-Pam2Cys against the M1 polarizing factors IFN- $\gamma$ and LPS on porcine macrophages. Differences in the modulation of surface marker expression (MHC I, MHC II, CD25, CD14, CD163, CD169) and cytokine release (IL-1 $\alpha$, IL-1 $\beta$, IL-6, IL-10, IL-12p40, TNF$\alpha)$ were assessed. MoM $\Phi$ stimulated with Mag-Pam2Cys presented a lower expression of CD25 and CD169 compared to moM1, in terms of both mean fluorescence intensity (MFI) and percentage of positive cells (Figure 5). MoM1 were also characterized by a higher expression of both MHC I and MHC II DR, although differences were statistically 
significant only for percentages of positive MHC II DR cells (Figure 5). Differences were observed in terms of CD14 expression: MoM1 presented a lower expression of this marker compared to macrophages stimulated with Mag-Pam2Cys (Figure 5).

a
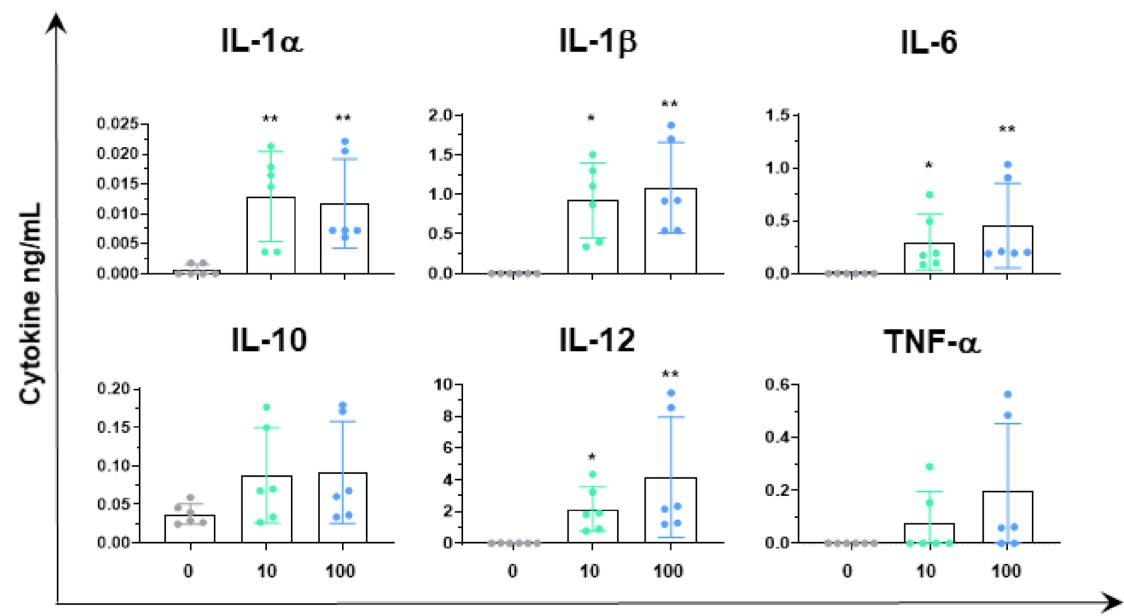

Mag-Pam2Cys (ng/mL)

b

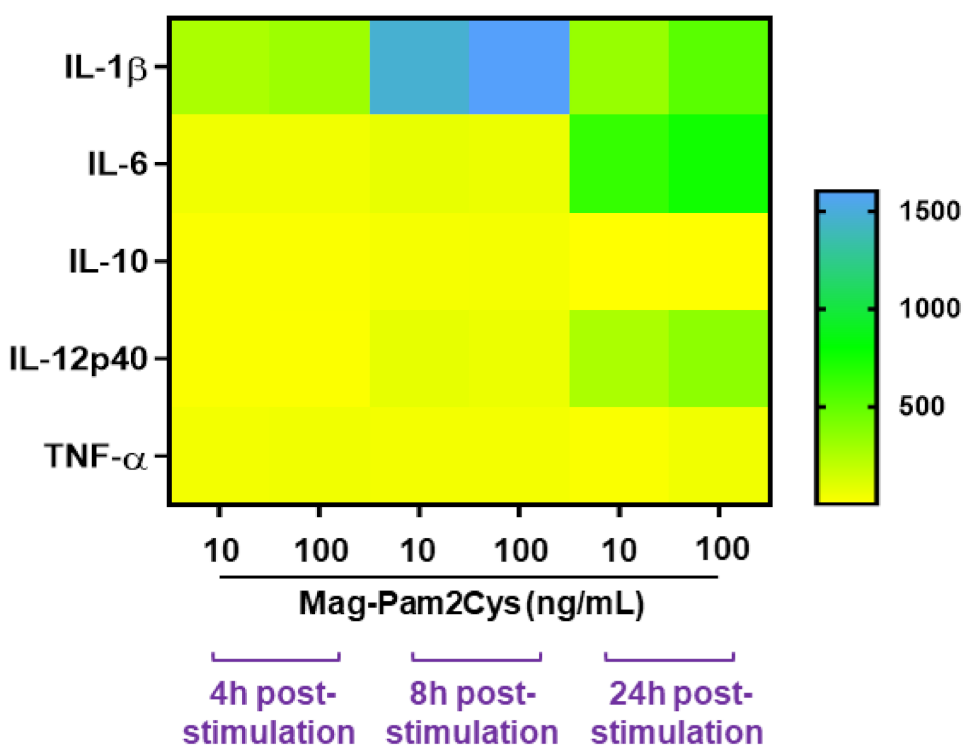

Figure 4. Mag-Pam2Cys' impact on cytokine induction and release. Porcine moM $\Phi$ were left untreated or stimulated with scalar doses of Mag-Pam2Cys (10 or $100 \mathrm{ng} / \mathrm{mL}$ ). (a) At $24 \mathrm{~h}$ poststimulation, levels of IL-1 $\alpha$, IL-1 $\beta$, IL-6, IL-10, IL-12, TNF- $\alpha$ in culture supernatants were quantified using a multiplex ELISA. Mean data and SD from three independent experiments using different blood donor pigs are presented. Values for Mag-Pam2Cys-stimulated samples were compared to the corresponding untreated control (moM $\Phi)$ using a Kruskal-Wallis multiple comparison test; ${ }^{* *} p<0.01$, ${ }^{*} p<0.05$. (b) At 4, 8, and 24 h post-stimulation, gene expression levels of IL-1 $\beta$, IL-6, IL-10, IL-12p40, TNF- $\alpha$ genes were determined using RT-qPCR. At each time point, data were normalized on the

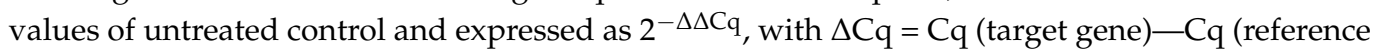
gene), and $\Delta \Delta \mathrm{Cq}=\Delta \mathrm{Cq}$ (Mag-Pam2Cys-stimulated samples) $-\Delta \mathrm{Cq}$ (untreated sample, moM $\Phi)$. Heatmap displays mean data from six independent experiments using different blood donor pigs. The colors in the cells represent the relative magnitude of gene expression. The green color represents the average magnitude of gene expression. The yellow color represents the smallest value, and the brightest blue represents the highest value. 
a

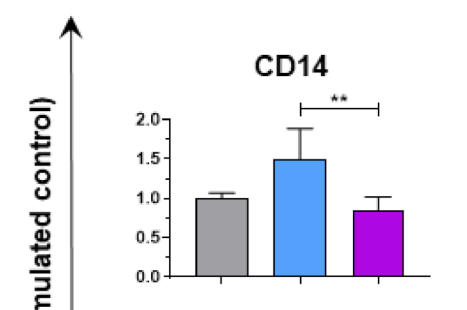

MHC II DR

CD25
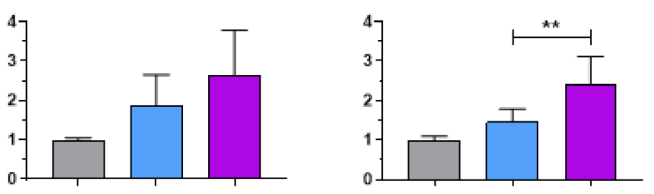

CD163

MHC I

CD169
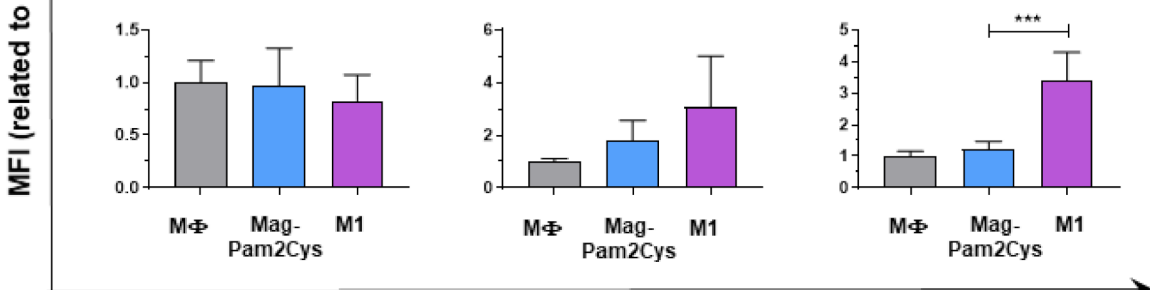

Treatment

b

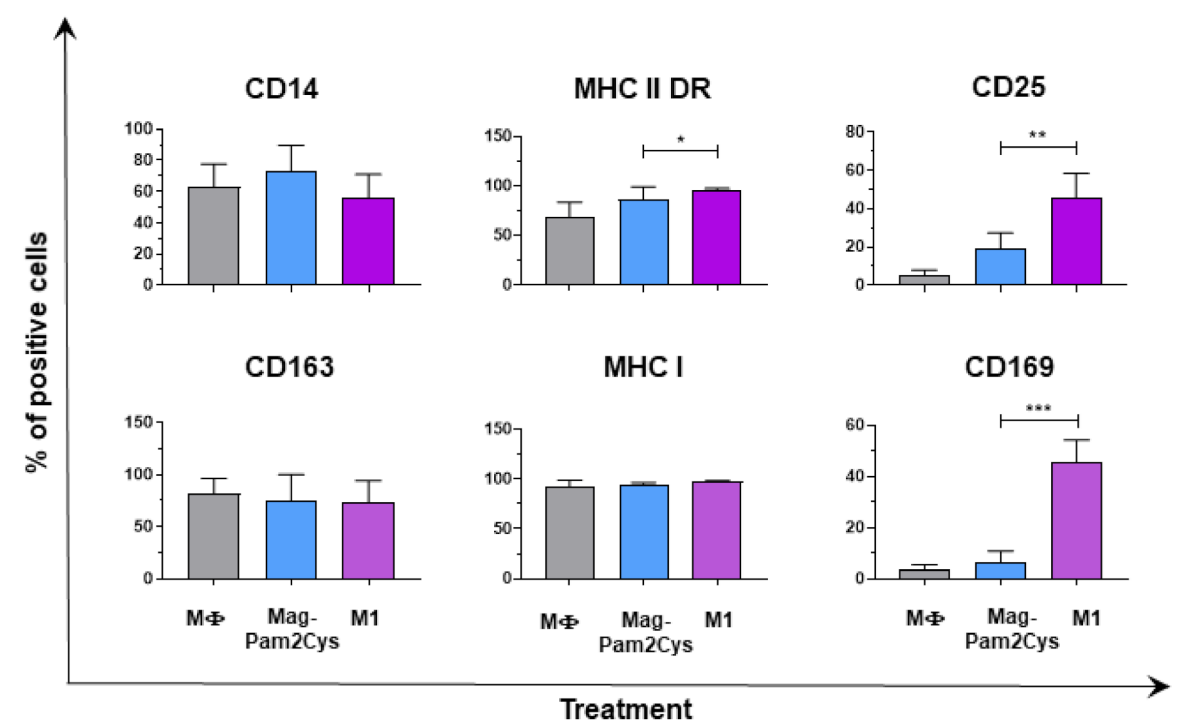

Figure 5. Modulation of moM $\Phi$ surface marker expressions by Mag-Pam2Cys and classical M1 polarizing factors. Porcine moM $\Phi$ were left untreated $(\mathrm{M} \Phi)$ or stimulated with Mag-Pam2Cys $(100 \mathrm{ng} / \mathrm{mL}$ ) or IFN- $\gamma+$ LPS (M1). At 24 h post-stimulation, surface expression of CD14, MHC II DR, CD25, CD163, MHC I, and CD169 were assessed by flow cytometry. In Panel (a), mean fluorescence intensity (MFI) data are presented as fold-change relative to the untreated control (moM $\Phi)$. In Panel (b), percentages of positive cells are shown. For both Panels, mean data and SD from three independent experiments using different blood donor pigs are displayed. Values of Mag-Pam2Cystreated samples were compared to bona fide moM1 (M1), using a Mann-Whitney test. ${ }^{* * *} p<0.001$, ** $p<0.01, * p<0.05$.

Stimulation of moM $\Phi$ with Mag-Pam2Cys or with IFN- $\gamma /$ LPS resulted in different patterns of cytokine release. MoM $\Phi$ stimulated with Mag-Pam2Cys presented a higher release of IL-6, IL-1 $\beta$, IL-1 $\alpha$ compared to moM1, although the latter cytokine was not statistically significant (Figure 6). MoM $\Phi$ stimulation with IFN- $\gamma /$ LPS resulted instead in a higher release of IL-12 compared to $100 \mathrm{ng} / \mathrm{mL}$ of MagPam2Cys (Figure 6). 


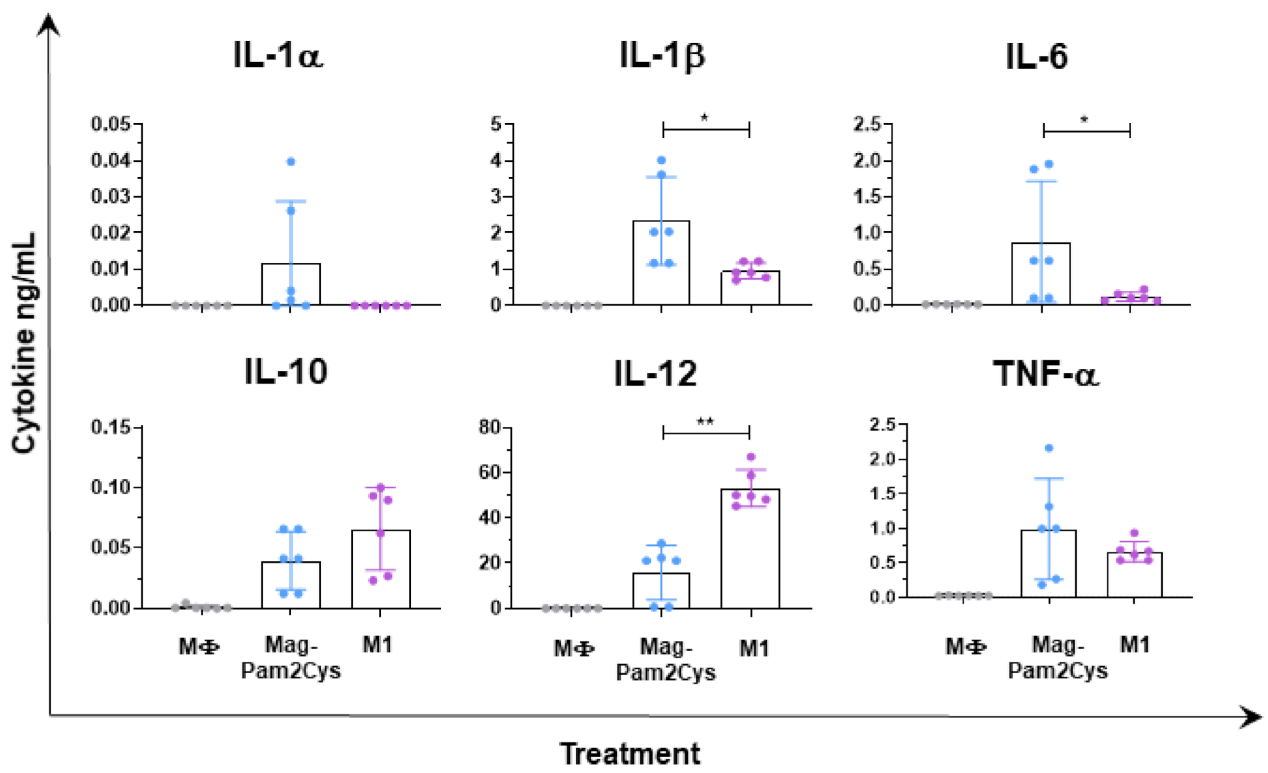

Figure 6. Cytokine release from moM $\Phi$ stimulated with Mag-Pam2Cys or IFN- $\gamma$ and LPS. Porcine moM $\Phi$ were left untreated (M $\Phi)$ or stimulated with Mag-Pam2Cys (100 ng/mL) or IFN- $\gamma+$ LPS (M1). At $24 \mathrm{~h}$ post-stimulation, levels of IL-1 $\alpha$, IL-1 $\beta$, IL-6, IL-10, IL-12, TNF- $\alpha$ in culture supernatants were quantified using a multiplex ELISA. Mean data and SD from three independent experiments using different blood donor pigs are displayed. Values of Mag-Pam2Cys-treated samples were compared to bona fide moM1 (M1), using a Mann-Whitney test. ${ }^{* *} p<0.01,{ }^{*} p<0.05$.

\subsection{Effect of Mag-Pam2Cys on Key Macrophage Regulatory Gene Expression}

To gain a broader view of the effects of Mag-Pam2Cys on moM $\Phi$, additional gene expression analyses were performed. MoM $\Phi$ were exposed to Mag-Pam2Cys (10 or $100 \mathrm{ng} / \mathrm{mL}$ ) or left untreated, and at 4, 8, and $24 \mathrm{~h}$ post-stimulation, expression analysis of a further 28 selected key immune genes was performed.

Several type I IFNs were first evaluated. An analysis of IFN- $\beta$ and IFN- $\alpha$ subtypes gene expression showed that Mag-Pam2Cys induced a statistically significant enhanced expression of IFN- $\alpha 1$, IFN- $\alpha 2$, IFN- $\alpha 5 / 6$, IFN- $\alpha 9$ at $8 \mathrm{~h}$ post-stimulation (Figure 7 , Figure S4). A small increase in IFN- $\alpha 7,-\alpha 9,-\alpha 12,-\alpha 13,-\alpha 14$, and $-\alpha 16$ gene expression was also observed at $24 \mathrm{~h}$ post-stimulation with both Mag-Pam2Cys doses, albeit with only the latter statistically significant (Figure 7, Figure S4).

Th expression of IFN- $\beta$ was not statistically significantly altered by Mag-Pam2Cys at any time post-stimulation (Figure 7, Figure S5). Interestingly, a marked decrease in IFN- $\alpha 3$ gene expression was observed 24 h post-stimulation with either 10 or $100 \mathrm{ng} / \mathrm{mL}$ of Mag-Pam2Cys (Figure 7, Figure S5).

Modulation on the expression of six TLRs was then evaluated. TLR4 and TLR5 are cellsurface TLRs which recognize bacterial lipopolysaccharide (LPS) or flagellin, respectively, whereas TLR3, TLR7, TLR8, TLR9 are located intracellularly and recognize nucleic acids derived from bacteria and viruses [1,2]. Treatment with either low or high doses of MagPam2Cys induced a statistically significant downregulation of TLR3 (at 8 and $24 \mathrm{~h}$ ), TLR4 (at 4 and 24 h), TLR5 (at 8 and 24 h), TLR7 (at 24 h), TLR8 (at 4, 8, and 24 h), TLR9 (at 8 and $24 \mathrm{~h}$ ) (Figure 8). 


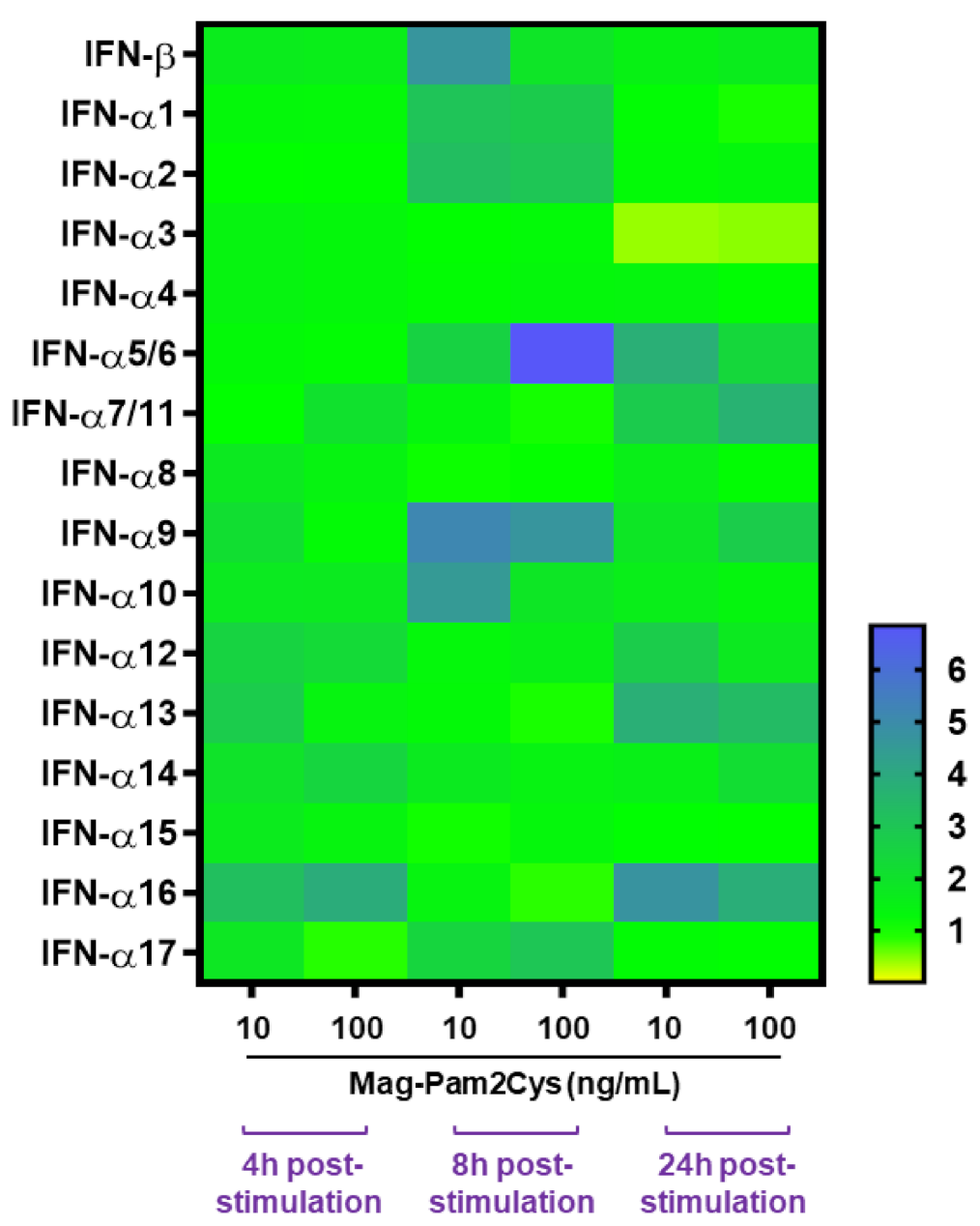

Figure 7. Heatmap of several type I IFNs genes differentially expressed in moM $\Phi$. Porcine moM $\Phi$ were left untreated or stimulated with scalar doses of Mag-Pam2Cys (10 or $100 \mathrm{ng} / \mathrm{mL}$ ). At 4, 8, and $24 \mathrm{~h}$ post-stimulation, gene expression levels of IFN- $\beta$ and several IFN- $\alpha$ subtypes $(-\alpha 1,-\alpha 2,-\alpha 3,-\alpha 4$, $-\alpha 5 / 6,-\alpha 7 / 11,-\alpha 8,-\alpha 9,-\alpha 10,-\alpha 11,-\alpha 12,-\alpha 13,-\alpha 14,-\alpha 15,-\alpha 16,-\alpha 17)$ genes were determined using qPCR. At each time point, data were normalized on the values of untreated control and expressed

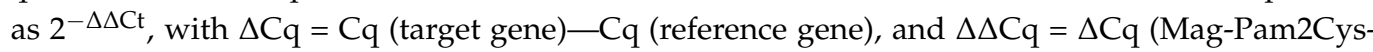
stimulated samples) $-\Delta \mathrm{Cq}$ (untreated sample, $\operatorname{moM} \Phi)$. Mean data from six independent experiments using different blood donor pigs are shown. The colors in the cells represent the relative magnitude of gene expression. The green color represents the average magnitude of gene expression. The yellow color represents the smallest value, and the brightest blue represents the highest value.

We next investigated the ability of Mag-Pam2Cys to modulate other key genes of innate immunity, such as the adaptor protein myeloid differentiation factor 88 (MYD88) and p65 (a subunit of transcription factor NF-kB), both involved in TLR signaling [43], and four molecules with antimicrobial properties: CD14 and myeloid differentiation factor 2 (MD2), both essential for the recognition of LPS by TLR4 [44], and the host antimicrobial peptides beta defensin 1 (BD1) and 2 (BD2), which exhibit antimicrobial activity against a broad range of bacteria [45-47]. The high dose $(100 \mathrm{ng} / \mathrm{mL})$ of Mag-Pam2Cys increased expression of p65 (at 4, 8 and 24 h), CD14 (at 8 h), and BD1 (at 8 h) with statistical significance. Enhanced expression of p65 was also observed in moM $\Phi$ treated with the low dose (10 ng/mL) of Mag-Pam2Cys (at 4 and $8 \mathrm{~h}$ ). MYD88 and MD2 expression were not altered by Mag-Pam2Cys at any tested concentration or timepoint (Figure 9). Finally, the effect of Mag-Pam2cys on the expression of nitric oxide synthase 2 (NOS2) was monitored, and we observed that both high and low doses of this TLR2 agonist enhanced NOS2 expression at all time points tested (Figure 9). 

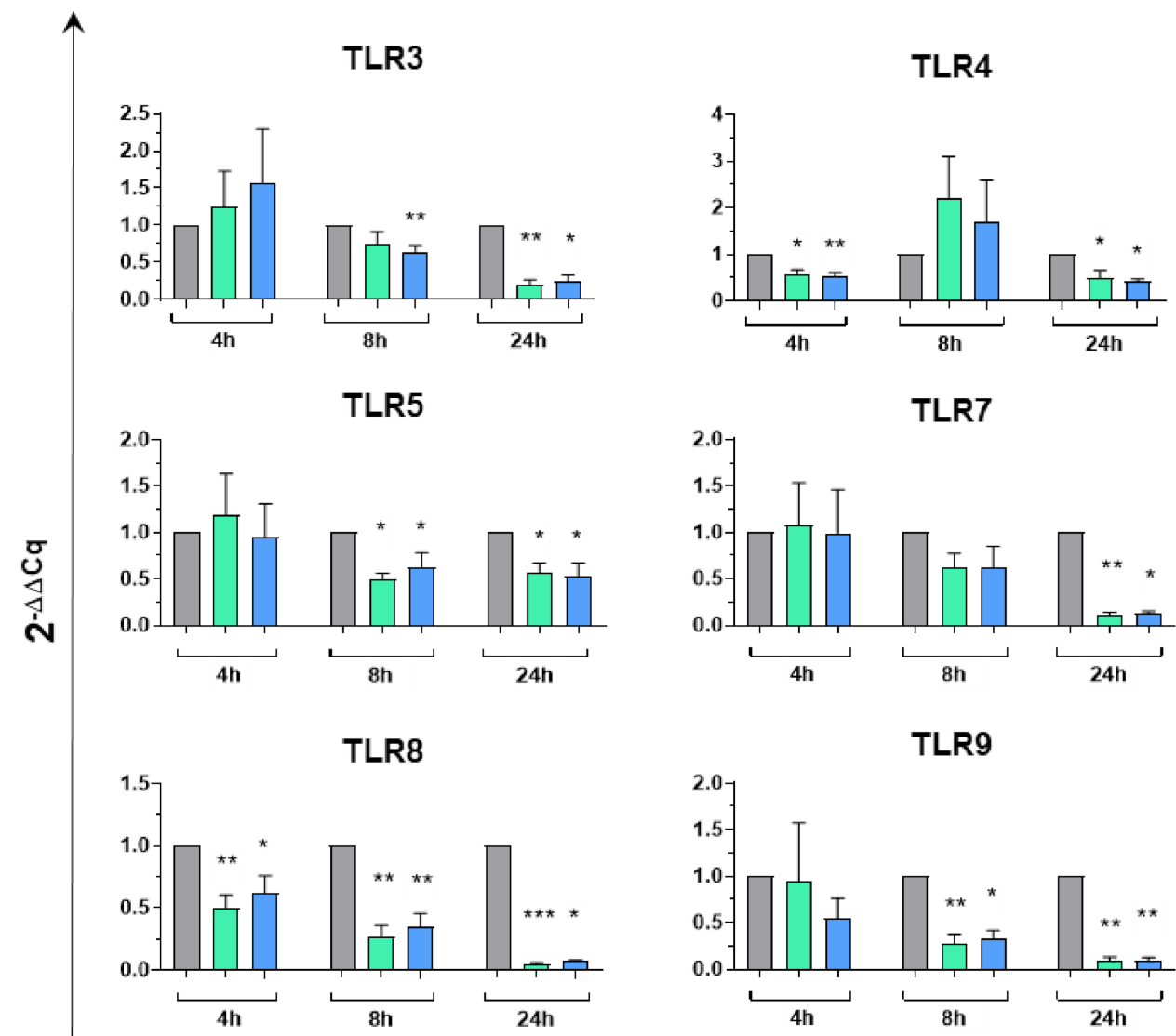

Untreated $\square 10 \mathrm{ng} / \mathrm{mL}$ Mag-Pam2Cys $\square 100 \mathrm{ng} / \mathrm{mL}$ Mag-Pam2Cys

\section{Treatment}

Figure 8. Toll-like receptors' (TLRs) gene expression in moM $\Phi$ following stimulation with MagPam2Cys. MoMФ were left untreated or stimulated with scalar doses of Mag-Pam2Cys (10 or $100 \mathrm{ng} / \mathrm{mL}$ ). At 4, 8, and $24 \mathrm{~h}$ post-stimulation, gene expression levels of six TLRs (TLR3, TLR4, TLR5, TLR7, TLR8, TLR9) were determined using qPCR. At each time point, data were normalized on the

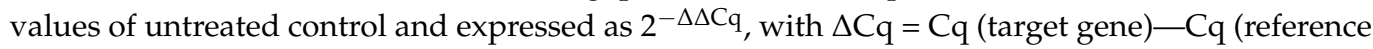
gene), and $\Delta \Delta \mathrm{Cq}=\Delta \mathrm{Cq}$ (Mag-Pam2Cys-stimulated samples) $-\Delta \mathrm{Cq}$ (untreated sample, moM $\Phi$ ). Mean data and SD from six independent experiments using different blood donor pigs are shown. For each time point, values of Mag-Pam2Cys stimulated samples were compared to the corresponding untreated control (moM $\Phi)$ using a Kruskal-Wallis multiple comparison test. ${ }^{* * *} p<0.001,{ }^{* *} p<0.01$, * $p<0.05$.

\subsection{Mag-Pam2Cys Ability to Affect moMФ Response to Recombinant Porcine IL-10}

To explore the effect of Mag-Pam2Cys on porcine moM $\Phi$ plasticity further, we evaluated its ability to modulate macrophage polarization induced by IL-10. We recently described that IL-10 polarizes porcine moM $\Phi$ towards an immunosuppressive phenotype (moM(IL-10)), characterized by a strong impairment of their ability to respond to either TLR2 or TLR4 agonists [19]. Porcine moM(IL-10) were also depicted by MHC II DR downregulation and enhanced surface expression of CD163 [19]. We evaluated whether Mag-Pam2Cys-induced activation impacted MHC II DR downregulation and CD163 upregulation triggered by recombinant porcine IL-10 (Figure 10). Treatment of $\mathrm{moM} \Phi$ with Mag-Pam2Cys either $24 \mathrm{~h}$ before (Figure 10a) or simultaneously (Figure 10b) with IL-10 treatment did not alter the downregulation of MHC II DR or upregulation CD163. 


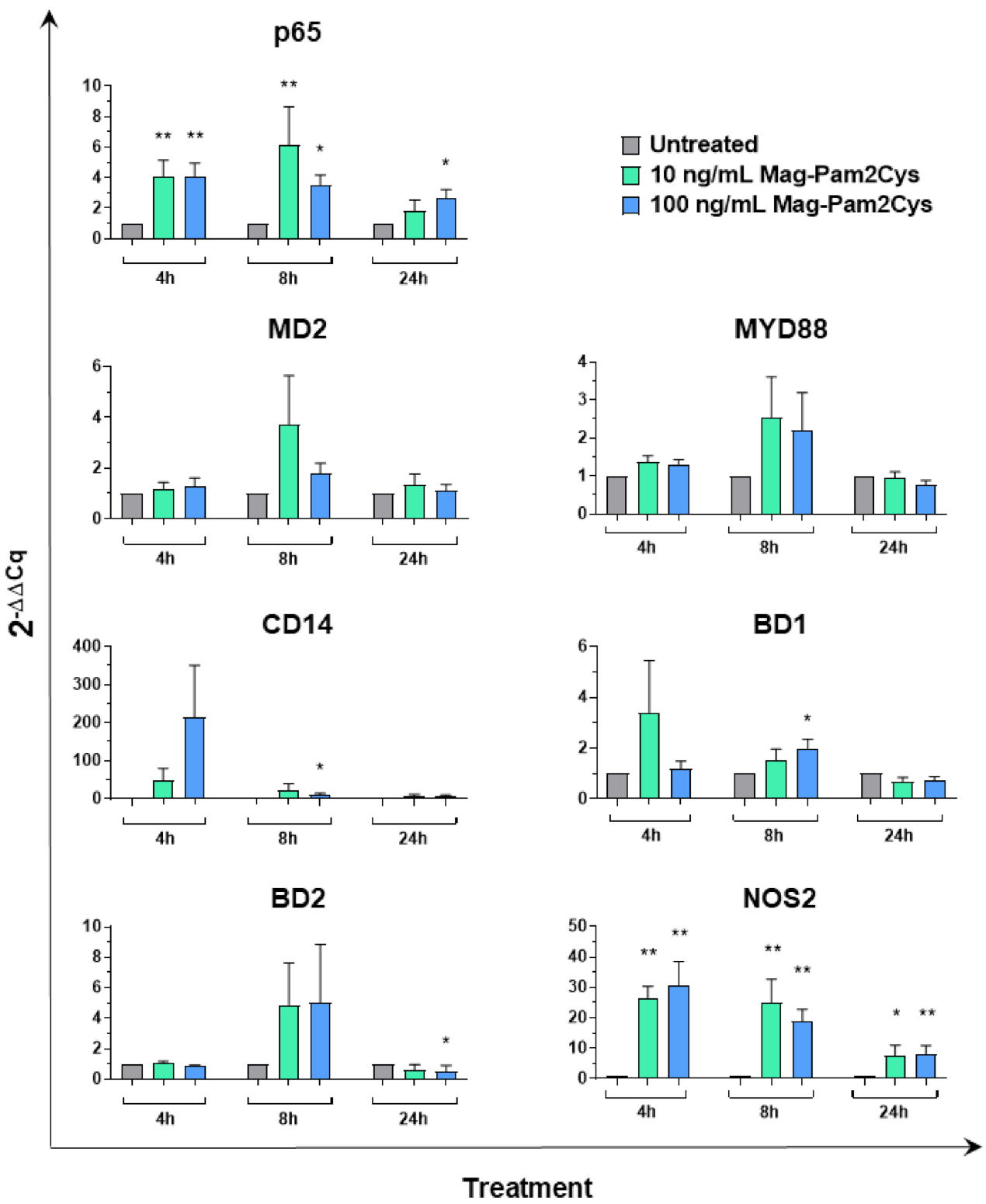

Figure 9. Gene expression of seven key genes of the innate immunity following stimulation with Mag-Pam2Cys. MoMФ were left untreated or stimulated with scalar doses of Mag-Pam2Cys (10 or $100 \mathrm{ng} / \mathrm{mL}$ ). At 4, 8, and $24 \mathrm{~h}$ post-stimulation, gene expression levels of p65, MYD88, MD2, CD14, BD1, BD2, NOS2 were determined using qPCR. At each time point, data were normalized on the

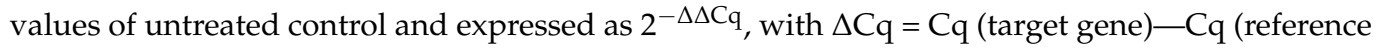
gene), and $\Delta \Delta \mathrm{Cq}=\Delta \mathrm{Cq}$ (Mag-Pam2Cys-stimulated samples) $-\Delta \mathrm{Cq}$ (untreated sample, moM $\Phi$ ). Mean data and SD from six independent experiments using different blood donor pigs are shown. For each time point, values of Mag-Pam2Cys-stimulated samples were compared to the corresponding untreated control $(\mathrm{moM} \Phi)$ using a Kruskal-Wallis multiple comparison test. ${ }^{* *} p<0.01,{ }^{*} p<0.05$. 
a

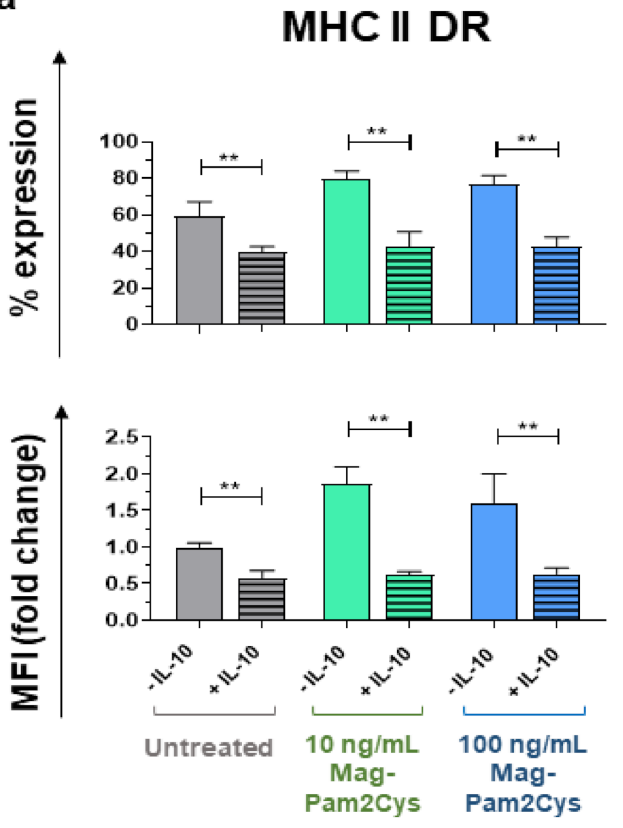

b

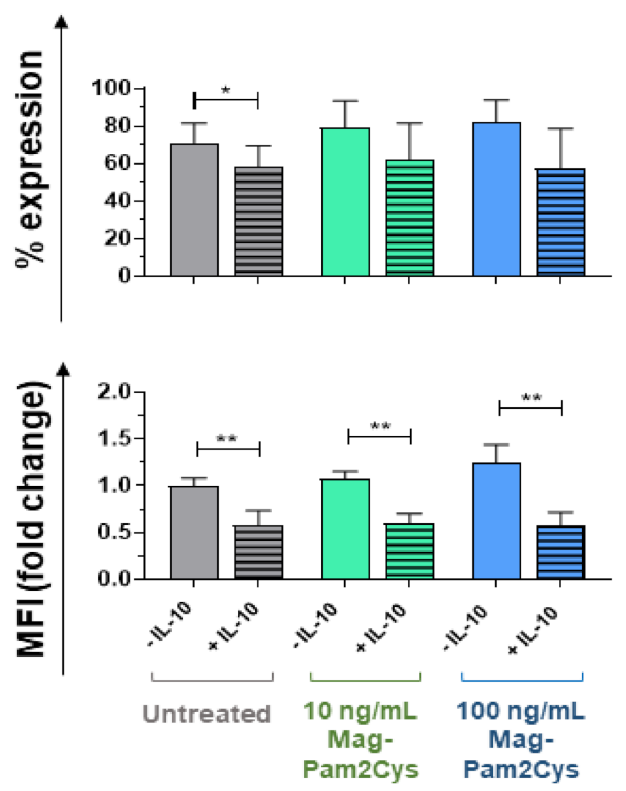

CD163
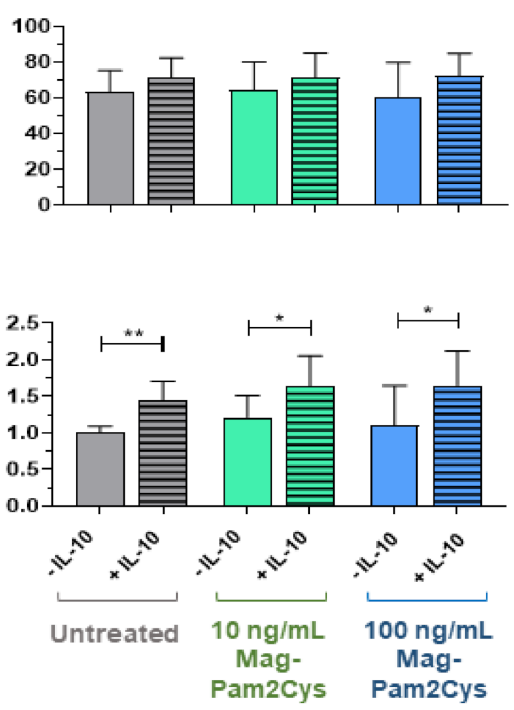

Figure 10. Mag-Pam2Cys modulation of $\operatorname{moM} \Phi$ response to IL-10 stimulation. Porcine moM $\Phi$ were left untreated or stimulated with scalar doses of Mag-Pam2Cys (10 or $100 \mathrm{ng} / \mathrm{mL}$ ). Simultaneously (a) or $24 \mathrm{~h}$ later $(\mathbf{b})$ recombinant porcine IL-10 $(20 \mathrm{ng} / \mathrm{mL})$ was added to well. Surface expression of MHC II DR and CD163 were evaluated by flow cytometry. Percentages of positive cells and mean fluorescence intensity (MFI) data (presented as fold-change relative to the untreated condition, moM $\Phi)$ are displayed in both panels. For both Panels $(\mathbf{a}, \mathbf{b})$, mean data and SD from three independent experiments using different blood donor pigs are presented. Values of stimulated samples were compared to the corresponding untreated control (moM $\Phi)$ using a one-way ANOVA followed by a Dunnett's multiple comparison test. ${ }^{* *} p<0.01,{ }^{*} p<0.05$.

\section{Discussion}

Macrophages play a central role in the innate immune response to both infectious and non-infectious stressors. They respond to different agonists modifying their phenotype and functions [48]. Macrophages polarized towards a M1 phenotype enhance 
defenses to intracellular pathogens and promote tumor regression, whereas alternatively activated macrophages (M2) are mainly associated with wound-healing and tissue repair [48]. Switching macrophages from a pro-tumor type (M2 phenotype) to an anti-tumor state (M1 phenotype) is a promising strategy in cancer immunotherapy [7]. Macrophages are equipped with a vast array of PRRs, including TLRs [21]. Among TLRs, TLR2 initiates potent immune responses by recognizing diacylated and triacylated lipopeptides, forming heterodimers with either TLR6 or TLR1, respectively [49]. We previously observed that Mag-Pam2Cys, a synthetic diacylated lipopeptide derived from a surface lipoprotein of $M$. agalactiae, induced a strong cytokine response from porcine $\operatorname{moM} \Phi[19,20]$; thus, in this study, we further investigated the genomic and functional impact of this lipopeptide on porcine $\mathrm{moM} \Phi$.

We observed that Mag-Pam2Cys enhanced the surface expression of molecules involved in antigen presentation (MHC class I and II) and the IL-2 receptor CD25, increased $\operatorname{moM} \Phi$ phagocytotic activities, and enhanced the expression and release of IL-12 and proinflammatory cytokines (IL- $1 \alpha$, IL-1 $\beta$, IL-6, TNF- $\alpha$ ) in a dose-dependent manner. Our results are in accordance with that previously observed in humans with MALP-2: the stimulation of human monocytes in vitro resulted in a sustained increased expression and release of cytokines (IL-6, TNF- $\alpha$ ) and chemokines (IL-8, GRO- $\alpha$, MCP-1, MIP-1 $\alpha$, and MIP-1 $\beta$ ) [50]. In the same study, a weak induction of the anti-inflammatory IL-10 was also observed [50], and this is in accordance with our results with porcine $\operatorname{moM} \Phi$. IL-10 induction or release are not hallmarks of M2 polarization in swine $[19,34]$ and it was previously reported by us and others that stimulation of porcine moM $\Phi$ with IFN- $\gamma$ and LPS (classical M1 activation stimuli) induced a low, but statistically significant, release of IL-10 [34,46]. Overall, our data suggest that Mag-Pam2Cys polarized porcine macrophages toward a M1-like phenotype, which in pigs is associated with increased expression of MHC I, MHC II DR, CD25, co-stimulatory molecules, and the release of proinflammatory cytokines [34,42,51].

To evaluate the polarizing effect of Mag-Pam2Cys on pig macrophages better, we directly compared the impact of a high dose $(100 \mathrm{ng} / \mathrm{mL})$ with the classical M1 polarizing factors (100 ng/mL IFN- $\gamma$ and $100 \mathrm{ng} / \mathrm{mL}$ LPS). MoM $\Phi$ stimulated with Mag-Pam2Cys presented a lower expression of CD25, CD169, MHC II DR and a higher expression of CD14 compared to bona fide moM1. MoM1 were characterized by a higher release of IL-12, whereas moM $\Phi$ stimulated with Mag-Pam2Cys triggered a higher release of several proinflammatory cytokines (IL- $1 \alpha$, IL-1 $\beta$, IL-6). These results highlight the plasticity of macrophages, which change their phenotype and function in response to different stimuli [48]. Indeed, it should be more appropriate to use a nomenclature linked to the activator(s) used instead of M1/M2, as suggested by Murray et al. [52]. To obtain a better understanding of the breadth of effects of Mag-Pam2Cys on porcine $\operatorname{moM} \Phi$, we analyzed its modulation of a panel of key innate immunity genes. Type I IFNs are a large family of antiviral proteins [53]. The porcine type I IFN family is complex and composed of at least 39 functional genes: one IFN- $\beta,-\alpha \omega,-\varepsilon$, and $-\kappa ; 17$ IFN- $\alpha$; 11 IFN- $\delta$; and 7 IFN- $\omega$ subtypes [54]. Despite their high structural homology, porcine IFN- $\alpha$ subtypes exhibit different anti-inflammatory, immunomodulatory, and antiviral activities against viruses, such as pseudorabies virus, PRRSV, classical swine fever virus (CSFV), and foot-and-mouth disease virus (FMDV) [53-56]. Thus, differences in the production of IFN- $\alpha$ subtypes might affect the endurance and intensity of an antiviral response. Mag-Pam2Cys induced a modest, but statistically significant, enhanced expression of IFN- $\alpha 1$, IFN- $\alpha 2$, IFN- $\alpha 5 / 6$, IFN- $\alpha 9$, and IFN- $\alpha 16$. These porcine IFNs exhibited direct antiviral activities towards many viruses, including PRRSV [54], CSFV [57], and pseudorabies [53], and presented immunomodulatory properties (inducing MHC I and MHC II upregulation) [53]. The induction of several type I IFNs, alongside proinflammatory cytokines, suggests that MagPam2Cys would promote host defense responses able to protect the host from microbial infection. However, the expression of IFN- $\beta$ and other IFN- $\alpha$ subtypes was not statistically significantly altered by Mag-Pam2Cys at any time points, and, most interestingly, a marked decrease in IFN- $\alpha 3$ gene expression was observed 24 h post-stimulation. Our results 
showed that type I IFNs were differently modulated upon stimulation with Mag-Pam2Cys. Further studies are needed to improve our understanding of the biological activities of individual type I IFNs and to appreciate fully how differences in their expression could influence the quality of antiviral responses.

We next specifically addressed how Mag-Pam2Cys modulated the expression of six TLRs and molecules involved in TLRs signaling. We observed that Mag-Pam2Cys treatment downregulated the expression of all the TLRs tested (TLR3, TLR4, TLR5, TLR7, TLR8, TLR9). Downregulation of TLR-4 and TLR8 was observed as soon as $4 \mathrm{~h}$ post-stimulation, whereas all the tested TLRs genes were drastically downregulated $24 \mathrm{~h}$ post-stimulation. A potential concern with stimulating macrophages through activation of TLRs is the induction of inappropriate inflammatory responses [3]; thus, the downregulated expression of other TLRs by Mag-Pam2Cys might present advantages in vivo. PAMP engagement by TLRs triggers several intracellular signaling cascades involving adapter molecule MyD88 and NF$\mathrm{KB}$, culminating in cytokine production and release $[3,6]$. The downregulated expression of these TLRs may represent protective mechanisms, considering that a tight regulation of this process is essential to avoid the development of pathological inflammatory responses or even autoimmunity [58].

The adaptor proteins MYD88 and the transcription factor NF- $\mathrm{kB}$ are both involved in the TLR2 intracellular signaling. Phosphorylation of p65 enhances the NF- $k B$ transcriptional response and thus induction of inflammatory cytokine genes [42]. We observed that this TLR2 agonist induced a strong increase in p65 gene expression, even at low doses, and this is in accordance with the observed enhanced expression and release of proinflammatory cytokines. Nevertheless, MYD88 expression was not modulated by Mag-Pam2Cys. We speculate that this null modulation of this adaptor protein might be the result of both positive and negative signals. As stated above, TLR signaling must be tightly regulated to prevent or terminate an excessive immune response. We then evaluated Mag-Pam2Cys impact on the gene expression of four molecules with antimicrobial properties: CD14 and MD2, BD1, and BD2 [43-46]. We observed that high doses of the diacylated lipopeptide under investigation increased the expression of either CD14 or BD1 with statistical significance, indicating that Mag-Pam2Cys stimulation triggered the enhancement of macrophage antimicrobial activities. Finally, modulation of NOS2 expression by this TLR2 agonist was evaluated. NOS2 encodes for the enzyme inducible nitric oxide synthase (iNOS), which generates nitic oxide (NO) from arginine [59]. NOS2 expression increased over time following Mag-Pam2Cys stimulation, further denoting the enhanced macrophage microbicidal functions promoted by this TLR2 agonist. Interestingly, iNOS expression is a hallmark of M1 polarization in many species [59], but not in swine. A previous study described that M1 polarization in pigs did not result in NO production or NOS2 upregulation [42], suggesting that future studies are needed to define macrophage polarization in pigs better.

Considering these observations, we speculate that administration of Mag-Pam2Cys in vivo might lead to macrophage activation with the release of proinflammatory cytokines, resulting in the recruitment of immune cells in situ and improving host defense against invading pathogens, as described in mice after intratracheal MALP-2 application or intranasal PEG-Pam2Cys administration [9,14]. In fact, intra-tracheal administration of MALP-2 to wild type (WT), but not TLR2-deficent, mice evoked enhanced proinflammatory cytokine and chemokine release, along with leukocyte recruitment into the lung [9]. Moreover, application of MALP-2 $24 \mathrm{~h}$ before intranasal infection with S. pneumonia resulted in increased survival, reduced bacteremia, and improved bacterial clearance in lung parenchyma in WT mice [9]. As mentioned above, intranasal administration of the synthetic PEG-Pam2Cys to mice led to reduced viral transmission rates following influenza infection, and promoted the development of adaptive immune responses, measured by the induction of influenza $\mathrm{A}$ virus-specific CD8+ T cells [14,15].

Overall, our results showed that Mag-Pam2Cys polarizes porcine macrophages toward a proinflammatory, antimicrobial phenotype $[42,60]$. Thus, we evaluated its ability to affect polarization triggered by the immunosuppressive IL-10 [21]. We recently described that 
IL-10 triggered MHC II DR downregulation and enhanced surface expression of CD163 on pig moM $\Phi$ [19]. Interestingly, Mag-Pam2Cys did not alter IL-10-induced MHC II DR downregulation or CD163 upregulation at any of the concentrations tested, either when administered $24 \mathrm{~h}$ before or in concomitance with porcine recombinant IL-10. The null interference of Mag-Pam2Cys with the activity of the immunosuppressive IL-10 stimulation is promising for the potential use of Mag-Pam2Cys as an immunotherapeutic agent, considering that IL-10 might regulate the development of exacerbated immune responses in vivo. Macrophages are characterized by remarkable plasticity [48]; thus, future studies should address how this TLR2 agonist affects macrophage polarization triggered by other immunosuppressive factors (as TGF- $\beta$ or glucocorticoids), or M2a factors (IL-4, IL-13), to evaluate its use as an immunotherapeutic agent better.

\section{Conclusions}

Herein, we have provided a detailed characterization of the effects of a synthetic diacylated mycoplasma-derived pam2cys lipopeptide, Mag-Pam2Cys, on porcine macrophages. We found that this TLR2 agonist induced enhanced expression of activation markers, increased phagocytotic activity, and the release of IL-12 and proinflammatory cytokines. Mag-Pam2Cys also induced expression of p65, CD14, BD1, NOS2, and several type I IFNs, indicating a polarization of macrophages towards a proinflammatory, antimicrobial phenotype. Nevertheless Mag-Pam2Cys negatively modulated TLR3, 4, 5, 7, 8, 9 gene expression and did not interfere with macrophage polarization induced by the immunosuppressive IL10 , suggesting that the inflammatory response induced by Mag-Pam2Cys can be regulated. This study lays the foundation for the further evaluation of this TLR-2 agonist in vivo as an immunomodulatory agent for pigs and other species.

Supplementary Materials: The following are available online at https:/ /www.mdpi.com/article/ 10.3390/vaccines9070692/s1, Figure S1. Study design. Figure S2. No morphological alterations of porcine moM following Mag-Pam2Cys stimulation. Figure S3. Representative dot plots of untreated or Mag-Pam2Cys-stimulated porcine $\mathrm{moM} \Phi$. Figure S4. Cytokine gene expression in moM $\Phi$ following stimulation with Mag-Pam2Cys. Figure S5. Type I IFN gene expression in moM $\Phi$ following stimulation with Mag-Pam2Cys. Table S1. Antibodies used for flow cytometry. Table S2. Oligonucleotide Primer Sets for Evagreen qRT Real-Time PCR.

Author Contributions: Conceptualization, G.F., B.C., E.R.; methodology, G.F., A.A., C.G.D.C., S.M., T.C., F.F., S.Z., G.V., S.D.G.; formal analysis, G.F., A.A., C.G.D.C., S.M., S.D.G., E.R.; investigation, G.F., A.A., C.G.D.C., S.M., T.C., F.F., S.Z., G.V., S.D.G.; data curation, G.F., A.A., S.P.G., E.R.; writing—original draft preparation, G.F.; writing—review and editing, G.F., S.P.G., B.C., E.R.; project administration, G.F., A.O., E.R.; funding acquisition, A.O., B.C., E.R. All authors have read and agreed to the published version of the manuscript.

Funding: This research was funded by the Italian Ministry of Health, grant IZSUM PSRC 1/2018, and grant IZS PLV 08/19 RC. S.P.G. is supported by an UKRI Biotechnology and Biological Sciences Research Council (BBSRC) Institute Strategic Programme Grant to the Pirbright Institute (BBS/E/I/00007031).

Institutional Review Board Statement: The present study was conducted according to the guidelines of the Declaration of Helsinki and approved by the Institutional Ethics Committee of the Istituto Zooprofilattico Sperimentale della Sardegna (authorization nº 1232/2020-PR).

Informed Consent Statement: Not applicable.

Data Availability Statement: The data presented in the study are available on request from the corresponding author.

Acknowledgments: The authors would like to acknowledge Paola Nicolussi, Giovannantonio Pilo, and all the Animal Services Unit (IZS della Sardegna, Italy) for the care of animals and provision of blood samples. The authors would also like to thank Pierpaolo Angioi (IZS della Sardegna, Italy), Gemma Bajardo (University of Sassary, Italy) for technical assistance during the experiments.

Conflicts of Interest: The authors declare no conflict of interest. 


\section{References}

1. Miyake, K. Innate immune sensing of pathogens and danger signals by cell surface Toll-like receptors. Semin. Immunol. 2007, 19, 3-10. [CrossRef] [PubMed]

2. Luchner, M.; Reinke, S.; Milicic, A. TLR Agonists as Vaccine Adjuvants Targeting Cancer and Infectious Diseases. Pharmaceutics 2021, 22, 142. [CrossRef] [PubMed]

3. Mifsud, E.; Tan, A.C.L.; Jackson, D.C. TLR agonists as modulators of the innate immune response and their potential as agents against infectious disease. Front. Immunol. 2014, 5, 79. [CrossRef] [PubMed]

4. Urban-Vojciuk, Z.; Khan, M.M.; Oyler, B.L.; Fåhraeus, R.; Marek-Trzonkowska, N.; Nita-Lazar, A.; Hupp, T.R.; Goodlett, D.R. The Role of TLRs in Anti-cancer Immunity and Tumor Rejection. Front. Immunol. 2019, 10, 2388. [CrossRef] [PubMed]

5. Gugliandolo, E.; Fusco, R.; Ginestra, G.; D’amico, R.; Bisignano, C.; Mandalari, G.; Cuzzocrea, S.; Di Paola, R. Involvement of TLR4 and PPAR- $\alpha$ Receptors in Host Response and NLRP3 Inflammasome Activation, Against Pulmonary Infection with Pseudomonas Aeruginosa. Shock 2019, 51, 221-227. [CrossRef] [PubMed]

6. Franchi, L.; Munoz-Planillo, R.; Nunez, G. Sensing and reacting to microbes through the inflammasomes. Nat. Immunol. 2012, 13, 325-332. [CrossRef] [PubMed]

7. Feng, Y.; Mu, R.; Wang, Z.; Xing, P.; Zhang, J.; Dong, L.; Wang, C. A toll-like receptor agonist mimicking microbial signal to generate tumor-suppressive macrophages. Nat. Commun. 2019, 10, 2272. [CrossRef] [PubMed]

8. Muhlradt, P.F.; Kiess, M.; Meyer, H.; Sussmuth, R.; Jung, G. Isolation, structure elucidation, and synthesis of a macrophage stimulatory lipopeptide from mycoplasma fermentans acting at picomolar concentration. J. Exp. Med. 1997, 185, 1951-1958. [CrossRef]

9. Reppe, K.; Tschernig, T.; Luhrmann, A.; Van Laak, V.; Grote, K.; Zemlin, M.V.; Gutbier, B.; Muller, H.C.; Kursar, M.; Schutte, H.; et al. Immunostimulation with macrophage-activating lipopeptide-2 increased survival in murine pneumonia. Am. J. Respir. Cell Mol. Biol. 2009, 40, 474-481. [CrossRef]

10. Zeng, W.; Ghosh, S.; Lau, Y.F.; Brown, L.E.; Jackson, D.C. Highly immunogenic and totally synthetic lipopeptides as selfadjuvanting immunocontraceptive vaccines. J. Immunol. 2002, 169, 4905-4912. [CrossRef]

11. Jackson, D.C.; Lau, Y.F.; Le, T.; Suhrbier, A.; Deliyannis, G.; Cheers, C.; Smith, C.; Zeng, W.; Brown, L.E. A totally synthetic vaccine of generic structure that targets Toll-like receptor 2 on dendritic cells and promotes antibody or cytotoxic T cell responses. Proc. Natl. Acad. Sci. USA 2004, 101, 15440-15445. [CrossRef]

12. Deliyannis, G.; Kedzierska, K.; Lau, Y.F.; Zeng, W.; Turner, S.J.; Jackson, D.C.; Brown, L.E. Intranasal lipopeptide primes lungresident memory CD8+ T cells for longterm pulmonary protection against influenza. Eur. J. Immunol. 2006, 36, 770-778. [CrossRef]

13. Chua, B.Y.; Zeng, W.; Jackson, D.C. Synthesis of toll-like receptor-2 targeting lipopeptides as self-adjuvanting vaccines. Methods Mol. Biol. 2008, 494, 247-261.

14. Tan, A.C.L.; Mifsud, E.J.; Zeng, W.; Edenborough, K.; Mcvernon, J.; Brown, L.E.; Jackson, D.C. Intranasal administration of the TLR2 agonist Pam2Cys provides rapid protection against influenza in mice. Mol. Pharm. 2012, 9, 2710-2718. [CrossRef]

15. Tan, A.C.L.; Eriksson, E.M.Y.; Kedzierska, K.; Deliyannis, G.; Valkenburg, S.A.; Zeng, W.; Jackson, D.C. Polyfunctional CD8+ T cells are associated with the vaccination-induced control of a novel recombinant influenza virus expressing an HCV epitope. Antiviral Res. 2012, 94, 168-178. [CrossRef]

16. Proud, P.C.; Tsitoura, D.; Watson, R.J.; Chua, B.Y.; Aram, M.J.; Bewley, K.R.; Cavell, B.E.; Cobb, R.; Dowall, S.; Fotheringham, S.A.; et al. Prophylactic intranasal administration of a TLR2/6 agonist reduces upper respiratory tract viral shedding in a SARS-CoV-2 challenge ferret model. EBioMedicine 2021, 63, 103153. [CrossRef]

17. Cacciotto, C.; Cubeddu, T.; Addis, M.F.; Anfossi, A.G.; Tedde, V.; Tore, G.; Carta, T.; Rocca, S.; Chessa, B.; Pittau, B.; et al. Mycoplasma lipoproteins are major determinants of neutrophil extracellular trap formation. Cell. Microbiol. 2016, 18, 1751-1762. [CrossRef]

18. Anfossi, A.G.; Coraduzza, E.; Bonelli, P.; Piras, I.; Cacciotto, C.; Puggioni, G.; Masia, M.; Alberti, A.; Pittau, M.; Chessa, B. Sintesi e valutazione delle proprietà immunomodulatorie di un lipopeptide diacilato derivato da Mycoplasma agalactiae. In Proceedings of the Simposio di Immunologia Veterinaria, Florence, Italy, 28 May 2014.

19. Carta, T.; Razzuoli, E.; Fruscione, F.; Zinellu, S.; Meloni, D.; Anfossi, A.; Chessa, B.; Dei Giudici, S.; Graham, S.P.; Oggiano, A.; et al. Comparative Phenotypic and Functional Analyses of the Effects of IL-10 or TGF- $\beta$ on Porcine Macrophages. Animals 2021, 11, 1098. [CrossRef]

20. Franzoni, G.; Razzuoli, E.; Dei Giudici, S.; Carta, T.; Galleri, G.; Zinellu, S.; Ledda, M.; Angioi, P.; Modesto, P.; Graham, S.P.; et al. Comparison of macrophage responses to African swine fever viruses reveals that the NH/P68 strain is associated with enhanced sensitivity to type I IFN and cytokine responses from classically associated macrophages. Pathogens 2020, 9, 209. [CrossRef]

21. Martinez, F.O.; Helming, L.; Gordon, S. Alternative activation of macrophages: An immunologic functional perspective. Annu. Rev. Immunol. 2009, 27, 451-483. [CrossRef]

22. Franzoni, G.; Dei Giudici, S.; Oggiano, A. Infection, modulation and responses of antigen-presenting cells to African swine fever viruses. Virus Res. 2018, 258, 73-80. [CrossRef] [PubMed]

23. Amadori, M.; Razzuoli, E. Immune control of PRRS: Lessons to be learned and possible ways forward. Front. Vet. Sci. 2014, 1, 1-14. [CrossRef] [PubMed]

24. Allan, G.M.; Ellis, J.A. Porcine circoviruses: A review. J. Vet. Diagn. Investig. 2000, 12, 3-14. [CrossRef] [PubMed]

25. VanderWaal, K.; Deen, J. Global trends in infectious diseases of swine. Proc. Natl. Acad. Sci. USA 2018, 115, 11495-11500. [CrossRef] 
26. Kapentanovic, R.; Fairbairn, L.; Beraldi, D.; Sester, D.P.; Archibald, A.L.; Tuggle, C.K.; Hume, D.A. Pig bone marrow-derived macrophages resemble human macrophages in their response to bacterial lipopolysaccharide. J. Immunol. 2012, 18, 3382-3394. [CrossRef]

27. Kapentanovic, R.; Fairbairn, L.; Downing, A.; Beraldi, D.; Sester, D.P.; Freeman, T.C.; Tuggle, C.K.; Archibald, A.L.; Hume, D.A. The impact of breed and tissue compartment on the response of pig macrophages to lipopolysaccharide. BMC Genom. 2013, 14,581 .

28. Fairbairn, L.; Kapetanovic, R.; Sester, D.P.; Hume, D.A. The mononuclear phagocyte system of the pig as a model for understanding human innate immunity and disease. J. Leukoc. Biol. 2011, 8, 855-871. [CrossRef]

29. Meurens, F.; Summerfield, A.; Nauwynck, H.; Saif, L.; Gerdts, V. The pig: A model for human infectious diseases. Trends Microbiol. 2012, 20, 50-57. [CrossRef]

30. Summerfield, A.; Meurens, F.; Ricklin, M.E. The immunology of the porcine skin and its value as a model for human skin. Mol. Immunol. 2015, 66, 14-21. [CrossRef]

31. Kaser, T. Swine as biomedical animal model for T-cell research-Success and potential for transmittable and non-transmittable human diseases. Mol. Immunol. 2021, 135, 95-115. [CrossRef]

32. Pabst, R. The pig as a model for immunology research. Cell Tissue Res. 2020, 380, 287-304. [CrossRef]

33. Franzoni, G.; Dei Giudici, S.; Loi, F.; Sanna, D.; Floris, M.; Fiori, M.; Sanna, M.L.; Madrau, P.; Scarpa, F.; Zinellu, S.; et al. African Swine Fever Circulation among Free-Ranging Pigs in Sardinia: Data from the Eradication Program. Vaccines 2020 8, 549. [CrossRef]

34. Franzoni, G.; Bonelli, P.; Graham, S.P.; Anfossi, A.G.; Dei Giudici, S.; Pilo, G.; Pittau, M.; Nicolussi, P.; Oggiano, A. Comparative phenotypic and functional analyses of the effects of autologous plasma and recombinant human macrophage-colony stimulating factor (M-CSF) on porcine monocyte to macrophage differentiation. Vet. Immunol. Immunopathol. 2017, 187, 80-88. [CrossRef]

35. Razzuoli, E.; Mignone, G.; Lazzara, F.; Vencia, W.; Ferraris, M.; Masiello, L.; Vivaldi, B.; Ferrari, A.; Bozzetta, E.; Amadori, M. Impact of cadmium exposure on swine enterocytes. Toxicol. Lett. 2018, 287, 92-99. [CrossRef]

36. Mecocci, S.; Porcellato, I.; Armando, F.; Mechelli, L.; Brachelente, C.; Pepe, M.; Gialletti, R.; Passeri, B.; Modesto, P.; Ghelardi, A.; et al. Equine Genital Squamous Cell Carcinoma Associated with EcPV2 Infection: RANKL Pathway Correlated to Inflammation and Wnt Signaling Activation. Biology 2021, 10, 244. [CrossRef]

37. Razzuoli, E.; Amadori, M.; Lazzara, F.; Bilato, D.; Ferraris, M.; Vito, G.; Ferrari, A. Salmonella serovar-specific interaction with jejunal epithelial cells. Vet. Microbiol. 2017, 207, 219-225. [CrossRef]

38. Razzuoli, E.; Villa, R.; Amadori, M. IPEC-J2 cells as reporter system of the anti-inflammatory control actions of interferon-alpha. J. Interferon Cytokine Res. 2013, 33, 597-605. [CrossRef]

39. Razzuoli, E.; Villa, R.; Sossi, E.; Amadori, M. Reverse Transcription Real-Time PCR for Detection of Porcine Interferon $\alpha$ and $\beta$ Genes. Scand. J. Immunol. 2011, 74, 412-418. [CrossRef]

40. Cheng, G.; Chen, W.; Li, Z.; Yan, W.; Zhao, X.; Xie, J.; Liu, M.; Zhang, H.; Zhong, Y.; Zheng, Z. Characterization of the porcine alpha interferon multigene family. Gene 2006, 382, 28-38. [CrossRef]

41. Yoo, I.; Han, J.; Lee, S.; Jung, W.; Kim, J.H.; Kim, Y.W.; Kim, H.J.; Hong, M.; Ka, H. Analysis of stage-specific expression of the toll-like receptor family in the porcine endometrium throughout the estrous cycle and pregnancy. Theriogenology 2019, 125, 173-183. [CrossRef]

42. Sautter, C.A.; Auray, G.; Python, S.; Liniger, M.; Summerfield, A. Phenotypic and functional modulations of porcine macrophages by interferons and interleukin-4. Dev. Comp. Immunol. 2018, 84, 181-192. [CrossRef]

43. Gilmore, T.D. Introduction to NF-kB: Players, pathways, perspectives. Oncogene 2006, 25, 6680-6684. [CrossRef]

44. Petersen, C.B.; Nygård, A.; Fredholm, M.; Aasted, B.; Salomonsen, J. Cloning, characterization and mapping of porcine CD14 reveals a high conservation of mammalian CD14 structure, expression and locus organization. Dev. Comp. Immunol. 2006, 31, 729-737. [CrossRef]

45. Elahi, S.; Buchanan, R.M.; Attah-Poku, S.; Townsend, H.G.; Babiuk, L.A.; Gerdts, V. The host defense peptide beta-defensin 1 confers protection against Bordetella pertussis in newborn piglets. Infect. Immun. 2006, 74, 2338-2352. [CrossRef]

46. Li, C.L.; Xu, T.T.; Chen, R.B.; Huang, X.X.; Zhao, Y.C.; Bao, Y.Y.; Zhao, W.; Zheng, Z. Cloning, expression and characterization of antimicrobial porcine beta defensin 1 in Escherichia coli. Protein Expr. Purif. 2013, 88, 47-53. [CrossRef]

47. Veldhuizen, E.J.; Rijnders, M.; Claassen, E.A.; van Dijk, A.; Haagsman, H.P. Porcine beta-defensin 2 displays broad antimicrobial activity against pathogenic intestinal bacteria. Mol. Immunol. 2008, 45, 386-394. [CrossRef]

48. Mosser, D.M.; Edwards, J.P. Exploring the full spectrum of macrophage activation. Nat. Rev. Immunol. 2008, 8, 958-969. [CrossRef]

49. Schenk, M.; Belisle, J.T.; Modlin, R.L. TLR2 looks at lipoproteins. Immunity 2009, 31, 847-849. [CrossRef]

50. Kaufmann, A.; Muhlradt, P.F.; Gemsa, D.; Sprenger, H. Induction of Cytokines and Chemokines in Human Monocytes by Mycoplasma fermentans-Derived Lipoprotein MALP-2. Infect. Immun. 1999, 67, 6303-6308. [CrossRef]

51. Singleton, H.; Graham, S.P.; Bodman-Smith, K.B.; Frossard, J.; Steinbachet, F. Establishing porcine monocyte-derived macrophage and dendritic cell systems for studying the interaction with PRRSV-1. Front. Microbiol. 2016, 7, 832. [CrossRef]

52. Murray, P.J.; Allen, J.E.; Biswas, S.K.; Fisher, E.A.; Gilroy, D.W.; Goerdt, S.; Gordon, S.; Hamilton, J.A.; Ivashkiv, L.B.; Lawrence, T.; et al. Macrophage activation and polarization: Nomenclature and experimental guidelines. Immunity 2014, 41, 14-20. [CrossRef] [PubMed] 
53. Zanotti, C.; Razzuoli, E.; Crooke, H.; Soule, O.; Pezzoni, G.; Ferraris, M.; Ferrari, A.; Amadori, M. Different biological activities of swine IFN- $\alpha$ subtypes. J. Interferon Cytokine Res. 2015, 35, 990-1002. [CrossRef]

54. Sang, Y.; Rowland, R.R.R.; Hesse, R.A.; Blecha, F. Differential expression and activity of the porcine type I interferon family. Physiol. Genom. 2010, 42, 248-258. [CrossRef]

55. Fernandez-Sainz, I.; Ramanathan, P.; O’Donnell, V.; Diaz-San Segundo, F.; Velazquez-Salinas, L.; Sturza, D.F.; Zhu, J.; de Jos Santos, T.; Borca, M.V. Treatment with interferon-alpha delays disease in swine infected with a highly virulent CSFV strain. Virology 2015, 483, 284-290. [CrossRef] [PubMed]

56. Chinsangaram, J.; Moraes, M.P.; Koster, M.; Grubman, M.J. Novel viral disease control strategy: Adenovirus expressing alpha interferon rapidily protects swine from foot-and-mouth disease. J. Virol. 2003, 77, 1621-1625. [CrossRef]

57. Sosan, O.; Graham, S.P.; Everett, H.; Crudgington, B.; Bodman-Smith, K.; Crooke, H. Differential antiviral effect of porcine interferon alpha subtypes on classical swine fever virus infection of porcine monocytes. Cytokine 2012, 59, 552. [CrossRef]

58. Kawasaki, T.; Kawai, T. Toll-like receptor signaling pathways. Front. Immunol. 2014, 5, 461. [CrossRef]

59. Rath, M.; Muller, I.; Kropf, P.; Closs, E.I.; Munder, M. Metabolism via arginase or nitric oxide synthase: Two competing arginine pathways in macrophages. Front. Immunol. 2014, 5, 532. [CrossRef]

60. Mosser, D.M. The many faces of macrophage activation. J. Leukoc. Biol. 2003, 73, 209-212. [CrossRef] 